\title{
Reversal of Osteoporotic Activity by Endothelial Cell-Secreted Bone-Targeting and Biocompatible Exosomes
}

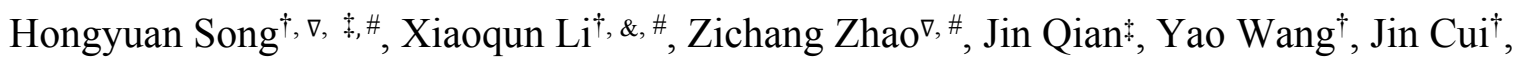
Weizong Weng ${ }^{\dagger}$, Liehu $\mathrm{Cao}^{\dagger}$, Xiao Chen ${ }^{\dagger}$, Yan $\mathrm{Hu}^{\dagger}$ and Jiacan $\mathrm{Su}^{\dagger}, *$

$\dagger$ Department of Orthopaedics Trauma, ${ }^{D}$ Department of Ophthalmology, Changhai Hospital, Second Military Medical University, Shanghai 200433, China

¥Department of Ophthalmology, Shanghai General Hospital (Shanghai First People's Hospital), School of Medicine, Shanghai Jiao Tong University 200080, Shanghai, China.

${ }^{\&}$ Graduate Management Unit, Shanghai Changhai Hospital, Second Military Medical University, Shanghai 200433, China

The $11^{\text {th }}$ Team of the $4^{\text {th }}$ Brigade of the Basic Medical Department, Second Military Medical University, Shanghai 200433, China

${ }^{*}$ Corresponding author:

Prof. Jiacan $\mathrm{Su}$

Department of Orthopaedics Trauma, Changhai Hospital, Second Military Medical University, Shanghai 200433, China

Emai: jiacansu@smmu.edu.cn

Tel: 862181873400

Fax: 862181873398

\#These authors contributed equally to this work. 


\section{Materials and Methods}

\section{Materials}

Fetal bovine serum (FBS), Dulbecco's minimum Eagle's medium (DMEM), Alexa Fluor ${ }^{\circledR} 555$ phalloidin, Trizol, and horse radish peroxidase-conjugated mouse and rabbit secondary antibodies were obtained from Thermo Fisher Scientific (Shanghai, China). The PrimeScript RT Reagent Kit, SYBR Premix Ex Taq, and Mir-X miRNA qRT-PCR SYBR ${ }^{\circledR}$ Kit were purchased from TaKaRa Biotechnology (Dalian, China). Anti- $\alpha$ tubulin antibody (Alexa Fluor® 647) was obtained from Abcam (Shanghai, China). Antibodies against Mtif, Spi1, Socs1, and GAPDH were obtained from Proteintech (Wuhan, China). The TRAP staining kit was obtained from Sigma-Aldrich (Shanghai, China). Dil staining reagent was purchased from Beyotime Biotechnology (Shanghai, China). Cell culturing plates and Osteo Assay Surface 96-Well Plates were purchased from Corning Inc. (Shanghai, China).

\section{Cell culture}

Mouse vascular endothelial cells (bEND.3) were purchased from KeyGEN BioTECH (Nanjing, China), and were cultured in DMEM supplemented with 10\% FBS, 1\% L-glutamine, $100 \mathrm{U} / \mathrm{mL}$ penicillin, and $100 \mathrm{mg} / \mathrm{mL}$ streptomycin. The cell lines were authenticated using the short tandem repeat method. The cells were incubated at $37^{\circ} \mathrm{C}$ in a humidified atmosphere with $5 \%$ $\mathrm{CO}_{2}$. The cells were negative for mycoplasma. When reaching $80-90 \%$ confluence, the cells were seeded into the appropriate plates for subsequent analysis.

\section{Isolation of exosomes}

We isolated exosomes from cell culture medium as described previously ${ }^{14}$. Briefly, any non-adherent cells in the medium were removed from the collected culture supernatant via centrifugation at $300 \times g$ for $10 \mathrm{~min}$ at $4^{\circ} \mathrm{C}$ followed by a second centrifugation at $800 \times g$ for 15 $\min$ and $10,000 \times g$ for $30 \mathrm{~min}$. The supernatant was ultracentrifuged (HITACHI, Japan) at $100,000 \times g$ for $70 \mathrm{~min}$. The pellet was washed with PBS to eliminate contaminated proteins and 
centrifuged at $100,000 \times g$ for another $70 \mathrm{~min}$. The protein concentration in the exosome suspension was determined using a BCA protein assay kit. Nanoparticle tracking analysis of exosomes was performed using ZetaView (Particle Metrix, Germany). The morphology of exosomes was observed by TEM (H-7650; Hitachi).

\section{Biophotonic imaging analysis}

C57BL/6 female mice were purchased from Slack Laboratory Animal Corp. (Shanghai, China). All animal studies were performed following protocols approved by the Laboratory Animal Center, Second Military Medical University, China. All animal procedures were carried out as regulated by the Second Military Medical University Animal Care and Use Committee. Mice were fed with alfalfa-free diet for 1 week before the experiment. Exosomes were labeled with Dil, and the labeled exosomes were intravenously injected into 8-week-old female C57BL/6 mice $(100 \mu \mathrm{g} / 100 \mu \mathrm{L}$ per mouse). The mice were killed at 4 and $8 \mathrm{~h}$ after injection and the relevant organs were excised. Finally, fluorescence imaging to determine the distribution of labeled exosomes was performed using a Quickview 3000 imaging system.

\section{Mass Spectrometry analysis}

The concentration of the eluted protein was determined using the BCA protein assay. $200 \mu \mathrm{g}$ protein were transferred to a new tube and the final volume was adjusted into $100 \mu \mathrm{L}$ with $8 \mathrm{M}$ urea. $4 \mu \mathrm{L} 1 \mathrm{M}$ DTT was added and the samples were incubated at $55^{\circ} \mathrm{C}$ for $1 \mathrm{~h}$. Afterwards, 10 $\mu \mathrm{L} 1 \mathrm{M}$ iodoacetamide was added and the samples were incubated for $1 \mathrm{~h}$ at room temperature in the dark. Then, 5 volumes of pre-chilled acetone were added to precipitate the proteins. The precipitates were washed twice with acetone, re-dissolved in $500 \mathrm{mM}$ TEAB (triethylammonium bicarbonate), and digested with sequence-grade, modified trypsin (Promega, Madison, WI) and lyophilized. 
The peptides were re-dissolved in $30 \mu \mathrm{L}$ solvent A (A: $0.1 \%$ formic acid in water) and analyzed by on-line nanospray LC-MS/MS on an Orbitrap Fusion coupled to an EASY-nano-LC 1200 system (Thermo Fisher Scientific, MA, USA). A $6 \mu \mathrm{L}$ peptide sample was loaded (trap column: Thermo Fisher Scientific Acclaim PepMap C18, $75 \mu \mathrm{m}$ x $2 \mathrm{~cm}$ ) and separated on the analytical column (Acclaim PepMap C18, $75 \mu \mathrm{m}$ x $50 \mathrm{~cm}$ ) with a linear gradient, from 3\% B (B: 0.1\% formic acid in $\mathrm{ACN}$ ) to $38 \% \mathrm{~B}$ in $120 \mathrm{~min}$. The column flow rate was maintained at $300 \mathrm{~nL} / \mathrm{min}$ with a column temperature of $40^{\circ} \mathrm{C}$. An electrospray voltage of $2 \mathrm{kV}$ versus the inlet of the mass spectrometer was used.

The mass spectrometer was run in data dependent acquisition mode, and automatically switched between MS and MS/MS mode. The parameters was as follows: (1) MS: scan range $(\mathrm{m} / \mathrm{z})=350$ 1650; resolution=70,000; $\mathrm{AGC}$ target $=3 \mathrm{e} 6$; maximum injection time $=50 \mathrm{~ms}$; include charge states $=2-7$; dynamic exclusion $=$ auto; (2) HCD-MS/MS: resolution $=17,500$; isolation window $=1.6 ;$ AGC target $=1 \mathrm{e} 5$; maximum injection time $=45 \mathrm{~ms}$; collision energy $=27$.

Tandem mass spectra were processed by PEAKS Studio version 8.5 (Bioinformatics Solutions Inc., Waterloo, Canada). PEAKS DB was set up to search the UniProt-human database (ver.201712, 72030 entries) assuming trypsin as the digestion enzyme. PEAKS DB was searched with a fragment ion mass tolerance of $0.05 \mathrm{Da}$ and a parent ion tolerance of $10 \mathrm{ppm}$. Carbamidomethylation (C) was specified as the fixed modification. Oxidation (M), Deamidation (NQ), Acetylation (Protein N-term), were specified as the variable modifications. Peptides were filtered by $1 \%$ FDR and 1 unique. PEAKSQ was used for peptide and protein abundance calculations. Normalization was performed by averaging the abundance of all peptides. Medians were used for averaging.

\section{Cellular uptake of EC-Exos by BMMs}

The fluorescent dye Dil (10 $\mu \mathrm{M}$, Beyotime, China) was added to EC-Exos suspensions, which 
were incubated for $20 \mathrm{~min}$ at room temperature. The stained exosomes were washed twice with PBS via ultracentrifugation at $100,000 \times g$ for $70 \mathrm{~min}$, and the pellet was resuspended in PBS. BMMs were incubated with the Dil-labeled EC-Exos for $2 \mathrm{~h}$. Then, the cells were washed twice with PBS and fixed in 4\% paraformaldehyde for $20 \mathrm{~min}$. After two washes with PBS, the cells were incubated with FITC- $\alpha$-tubulin and DAPI for $30 \mathrm{~min}$. Finally, the samples were analyzed using a confocal microscope (LSM-710; Zeiss, Germany).

\section{Cell morphology assay}

Approximately $1 \times 10^{4}$ BMMs were seeded into each well of a 96-well plate and cultured overnight. Then, various concentrations of EC-Exos were added to the cell culture medium and incubated for 2 days. The morphology of BMMs was examined using a digital microscope system (IX81; Olympus, Tokyo, Japan).

\section{In vitro osteoclastogenesis assay}

We isolated bone marrow cells from C57BL/6 mouse femur bones, and they were induced to BMMs using $25 \mathrm{ng} / \mathrm{mL}$ M-CSF for 3 days. Approximately $1 \times 10^{4}$ cells were seeded into each well of a 96-well plate and cultured overnight. Then, $25 \mathrm{ng} / \mathrm{mL}$ M-CSF and $100 \mathrm{ng} / \mathrm{mL}$ RANKL were applied to induce osteoclasts with or without treatment with various concentrations of exosomes. After 7 days, TRAP staining (Sigma-Aldrich, St. Louis, MO, USA) was performed following the manufacturer's instructions. TRAP-positive cells with greater than three nuclei were regarded as osteoclasts. Osteoclastogenic ability was determined by analyzing the TRAP-positive area compared with the total area.

\section{Actin ring formation assay}

Approximately $1 \times 10^{4}$ cells were seeded into each well of a 96-well plate and cultured overnight. Then, $25 \mathrm{ng} / \mathrm{mL}$ M-CSF and $100 \mathrm{ng} / \mathrm{mL}$ RANKL were applied to induce osteoclasts with or without treatment with various concentrations of exosomes. After 7 days, the cells were 
fixed in 4\% paraformaldehyde for $15 \mathrm{~min}$ at room temperature. Cells were washed at least four times with PBS and stained with Alexa Fluor 555 phalloidin for $1 \mathrm{~h}$, followed by DAPI staining for 20 min. Finally, the cells were observed using a digital microscope system (IX81; Olympus, Tokyo, Japan).

\section{Pit formation assay}

The pit formation assay was performed as we described previously. ${ }^{1}$ Approximately $1 \times 10^{4}$ cells were seeded into each well of a Corning® Osteo Assay Surface 96-well Plate and cultured overnight. Then, $25 \mathrm{ng} / \mathrm{mL}$ M-CSF and $100 \mathrm{ng} / \mathrm{mL}$ RANKL were applied to induce osteoclasts with or without treatment with various concentrations of exosomes. After 7 days, the culture medium was discarded and the surface was washed with $0.5 \%$ sodium hypochlorite. The plate was then washed twice with PBS and left to dry at room temperature for $3 \mathrm{~h}$. Finally, the resorbing area was imagaed under a digital microscope system and analyzed with ImageJ software.

\section{Co-culture assay using Transwell}

Approximately $1.2 \times 10^{5}$ BMMs were seeded into each well of a 24 -well plate and cultured overnight. Then, $25 \mathrm{ng} / \mathrm{mL}$ M-CSF and $100 \mathrm{ng} / \mathrm{mL}$ RANKL were added into the culture medium ( $\alpha$-MEM) to induce osteoclasts. Meanwhile, about $1 \times 10^{4}$ bEND.3 were seeded into Transwell inserts (polycarbonate membrane insert, $0.4 \mu \mathrm{m}$ pore; Corning Inc.) and cultured with $\alpha$-MEM overnight. Then, the inserts with bEND.3 were setting on the 24-well plate and co-cultured with BMMs for about 7 days. Thereafter, TRAP staining (Sigma-Aldrich, St. Louis, MO, USA) was performed following the manufacturer's instructions. For pit formation, BMMs were seeded into each well of a Corning® Osteo Assay Surface 24-well multiple well plates, and co-cultured with bEDN.3 as before.

\section{Animal model}


We created the ovariectomized mouse model in a pathogen free animal laboratory as we described previously. ${ }^{1-2}$ All animal studies were performed following protocols approved by the Laboratory Animal Center, Second Military Medical University, China. All animal procedures were carried out as regulated by the Second Military Medical University Animal Care and Use Committee. Eight-week-old female C57BL/6 mice were divided into three groups (9 mice each group): sham, ovariectomy, and treatment. For the EC-Exos-treated group, $100 \mu \mathrm{g}$ exosomes in $100 \mu \mathrm{L}$ PBS was intraperitoneally injected twice a week for 6 weeks. For the ovariectomy group, $100 \mu \mathrm{L}$ PBS was injected intraperitoneally twice a week for 6 weeks. We isolated bone marrow cells from femur bones, induced them to BMMs using $25 \mathrm{ng} / \mathrm{mL}$ M-CSF for 3 days. Then the expression of miR-155, Spi1, Mtif and Socs1 were detected using qRT-PCR and western blot. Meanwhile, femoral and arterial blood were collected for further experiments. The blood was centrifuged for $5 \mathrm{~min}$ at $1000 \mathrm{~g}$ and the supernatant was stored at $-80^{\circ} \mathrm{C}$. The femurs were used for further analysis.

\section{Bone histomorphometric analysis}

For the histological observations, we fixed the femur bone samples for 3 days in $4 \%$ paraformaldehyde and decalcified them for 20 days with $10 \%$ tetracycline-EDTA. Each distal femur was sliced into 4- $\mu \mathrm{m}$ sections and subjected to hematoxylin and eosin staining and TRAP staining. We calculated the trabecular bone area and number of osteoclasts within selected areas using ImageJ software.

\section{Microcomputed tomography analysis}

We analyzed 100 section planes from the growth plate of each bone (Skyscan 1172; Bruker microCT, Antwerp, Belgium). The metaphyseal region and trabecular bone were detected with built-in software. Bone mineral density, bone volume/total volume, bone surface area/total volume, and trabecular number were collected. We reconstructed the bone structure image slices into three-dimensional images. 


\section{Gene sequencing assay}

Total RNA was extracted using Trizol according to the manufacturer's instructions. The samples were sent to The Beijing Genomics Institute (Shenzhen, China) for further analysis. Differentially expressed genes were identified via fold change filtering (the minimum change considered at "hit" is two-fold). Multi Experiment Viewer was used for hierarchical clustering based on the average linkage.

\section{Real-time PCR}

miR-155 mimics and inhibitor were synthesized by RiboBio Co., Ltd. (Guangzhou, China). Total RNA was extracted using Trizol according to the manufacturer's instructions. The concentration and purity of RNA were estimated by measuring the optical density at $260 \mathrm{~nm} / 280 \mathrm{~nm}$. Equal quantities of RNA were transcribed into cDNAs using a PrimeScript RT Reagent Kit. Then,

equal quantities of cDNA were subjected to qRT-PCR using SYBR, while U6 was used as the control. Primers used for Real time PCR are shown in Table S4.

\section{Western blotting}

BMMs $\left(4 \times 10^{5}\right.$ cells per well $)$ were seeded into six-well plates and cultured overnight. The cells were then treated with different concentrations of exosomes for 2 days. Total protein was extracted in RIPA lysis buffer $(50 \mathrm{mM}$ Tris, $150 \mathrm{mM} \mathrm{NaCl}, 1 \% \mathrm{NP}-40$ and $0.5 \%$ sodium deoxycholate) with a proteinase inhibitor cocktail. Next, $10 \mu \mathrm{g}$ protein was subjected to SDS-PAGE and transferred onto polyvinylidene fluoride membranes. After blocking with nonfat milk for $2 \mathrm{~h}$, the membranes were incubated with the indicated primary antibodies at $4^{\circ} \mathrm{C}$ overnight. Next, the blots were washed three times with TBS-T and incubated with secondary antibodies for $2 \mathrm{~h}$ at room temperature. After three washes with TBS-T, the membranes were incubated in chemiluminescent substrate and visualized using the GeneGnome HR Image Capture System. 


\section{Statistical analysis}

All experiments were performed at least three times and are presented as the mean \pm standard deviation. One-way analysis of variance for three groups and Student's $t$-test for two groups were performed for the statistical analysis using GraphPad Prism 5.0 (GraphPad Software). A value of $p<0.05$ was considered statistically significant. 

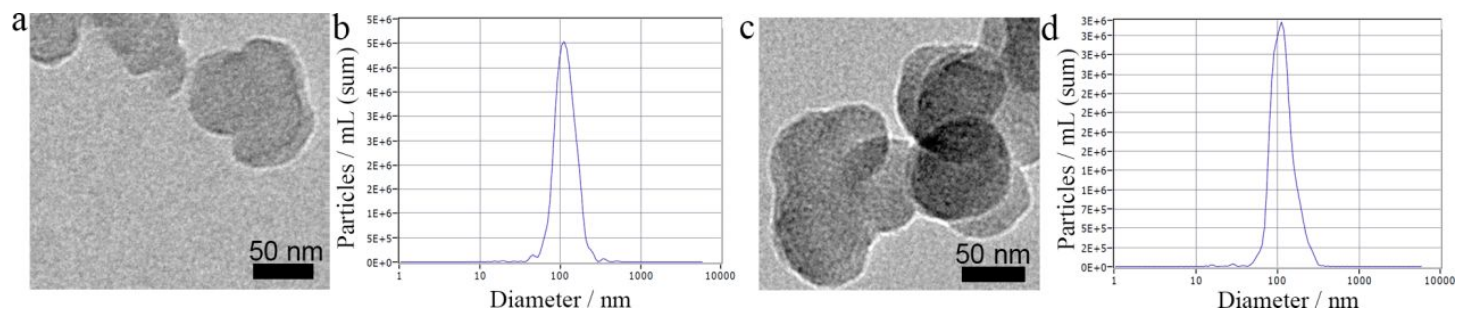

Figure S1. Characteristics of isolated exosomes. (a) Representative TEM image of BMSCs-Exos (scale bar $50 \mathrm{~nm}$ ). (b) Representative result of NTA shows the size distribution of BMSCs-Exos. (c) Representative TEM image of MC3T3-Exos (scale bar $50 \mathrm{~nm}$ ). (d) Representative result of NTA shows the size distribution of MC3T3-Exos.

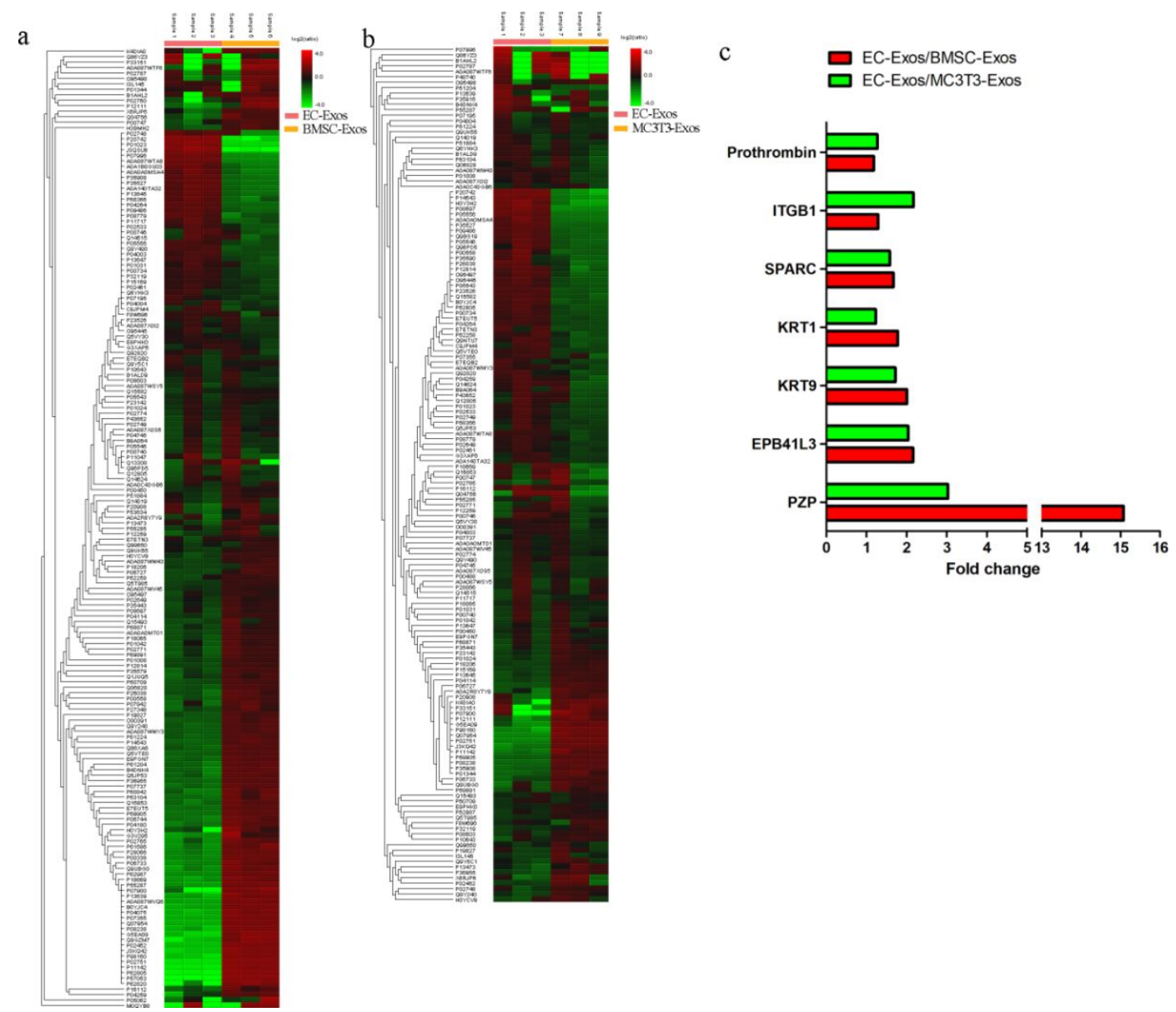

Figure S2. Differentially expressed exosomal proteins as determined by mass spectrometry. (a) Heatmap of the hierarchical clustering of differentially expressed proteins. Values represent the $\log _{2}$ fold change of proteins in EC-Exos compared with BMSC-Exos. Green and red colors indicate lower expression and higher expression, respectively. (b) Heatmap of the hierarchical clustering of differentially expressed proteins. Values represent the $\log _{2}$ fold change of proteins in EC-Exos compared with MC3T3-Exos. Green and red colors indicate lower expression and 
higher expression, respectively. (c) Proteins that were more highly expressed in EC-Exos compared with the other two types of exosomes.

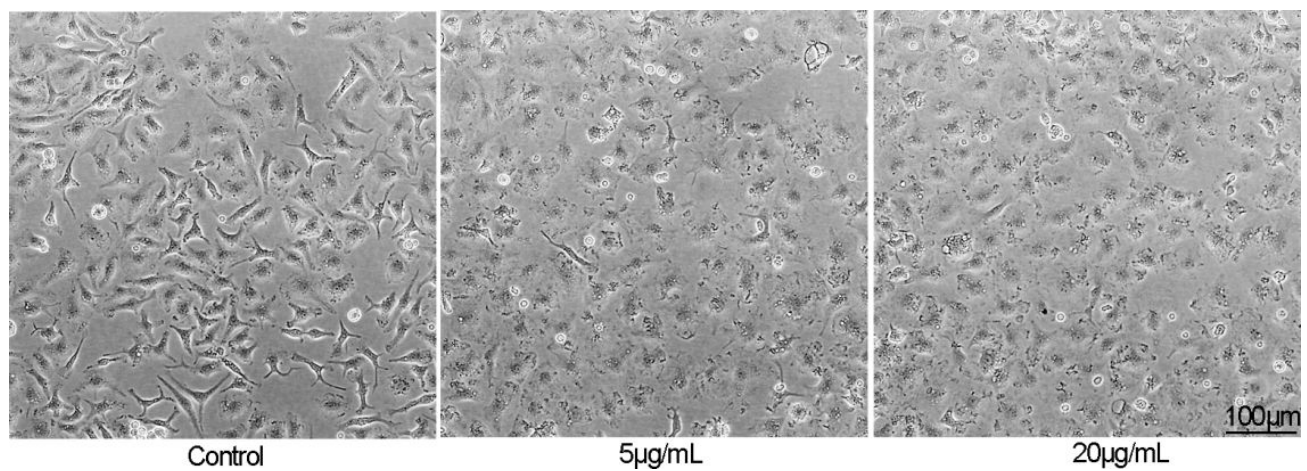

Figure S3. EC-Exos alter BMMs morphology. EC-Exos treatment $(5 \mu \mathrm{g} / \mathrm{mL}, 20 \mu \mathrm{g} / \mathrm{mL})$ changed the morphology of BMMs from spindle and round-shaped with good contrast (control) to round-shaped with poor contrast $($ scale bar $=100 \mu \mathrm{m})$.

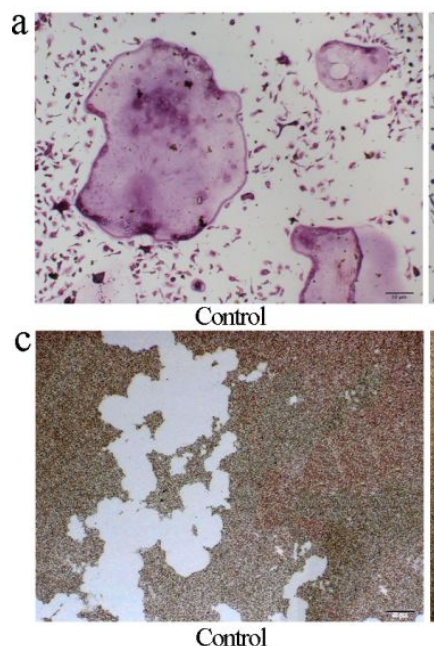

Control

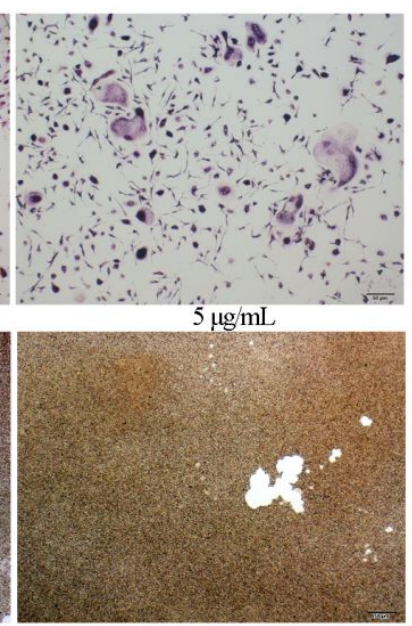

$5 \mu \mathrm{g} / \mathrm{mL}$
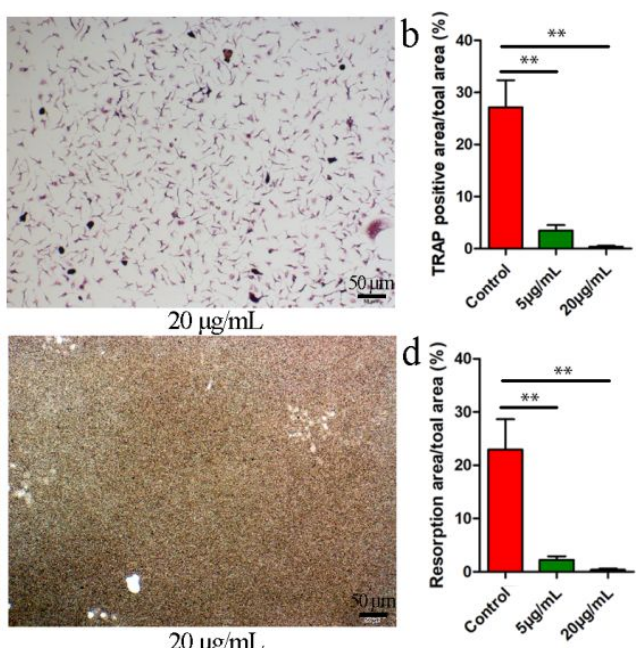

Figure S4. EC-Exos inhibited RAW264.7 differentiation, activation and bone resorption in vitro. (a) Representative images of TRAP-positive cells (scale bar $200 \mu \mathrm{m}$ ). (b) Quantitative analysis of the area of TRAP-positive osteoclasts. (c) Representative images of bone resorption by osteoclasts (scale bar $50 \mu \mathrm{m}$ ). (d) Quantitative analysis of the area of pits. Data are presented as the mean $\pm \mathrm{SD},\left({ }^{*} p<0.05,{ }^{* *} p<0.01\right)$. 

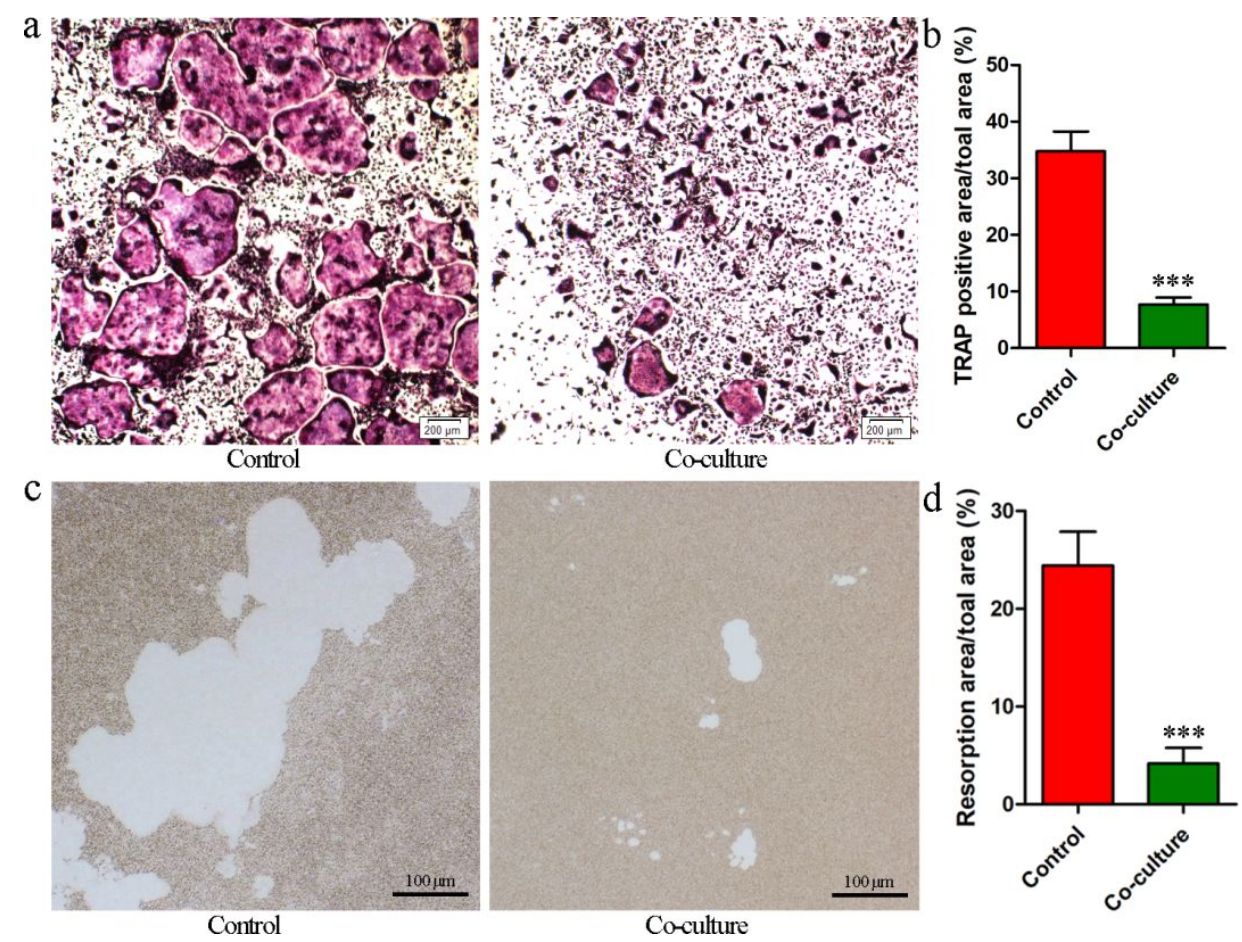

Figure S5. EC-Exos inhibited BMMs differentiation and bone resorption using co-culture system. (a) Representative images of TRAP-positive cells (scale bar $200 \mu \mathrm{m}$ ). (b) Quantitative analysis of the area of TRAP-positive osteoclasts. (c) Representative images of bone resorption by osteoclasts (scale bar $100 \mu \mathrm{m}$ ). (d) Quantitative analysis of the area of pits. Data are presented as the mean $\pm \mathrm{SD},(* * * p<0.001)$.
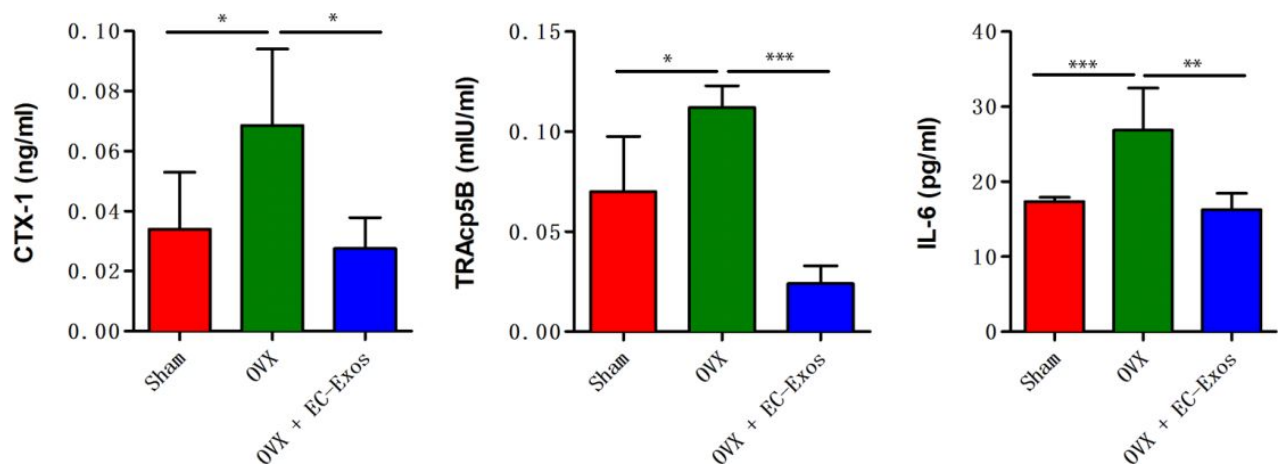

Figure S6. The concentrations of CTX-1, TRAcp5B and IL-6 in serum from sham operated, ovariectomized (OVX), and OVX with EC-Exos treatment mice were determined by enzyme-linked immunosorbent assay (ELISA). Data are presented as the meas $\pm \mathrm{SD},\left({ }^{*} p<0.05\right.$, $* * p<0.01, * * * p<0.001)$. 


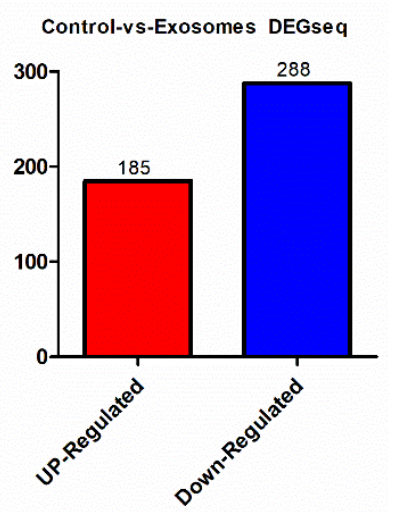

Figure S7 Statistical analysis of differentially expressed miRNAs. There were 473 miRNAs with different expression in total, including 185 up-regulated miRNAs and 288 down-regulated miRNAs (2-fold change, adjusted $p$-value, 0.01 ).

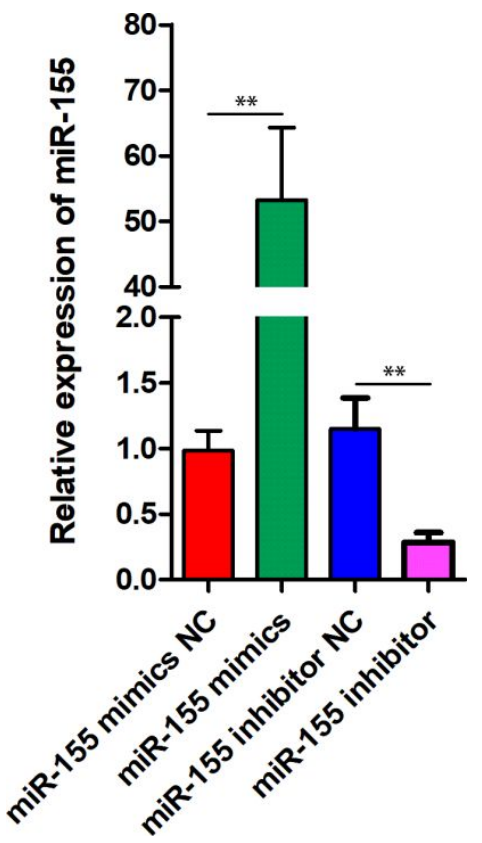

Figure S8. The expression of miR-155 in the miR-155 mimic negative control group, miR-155 mimic group, miR-155 inhibitor negative control group and miR-155 inhibitor group. Data are presented as the mean $\pm \mathrm{SD},(* p<0.05, * * p<0.01)$. 


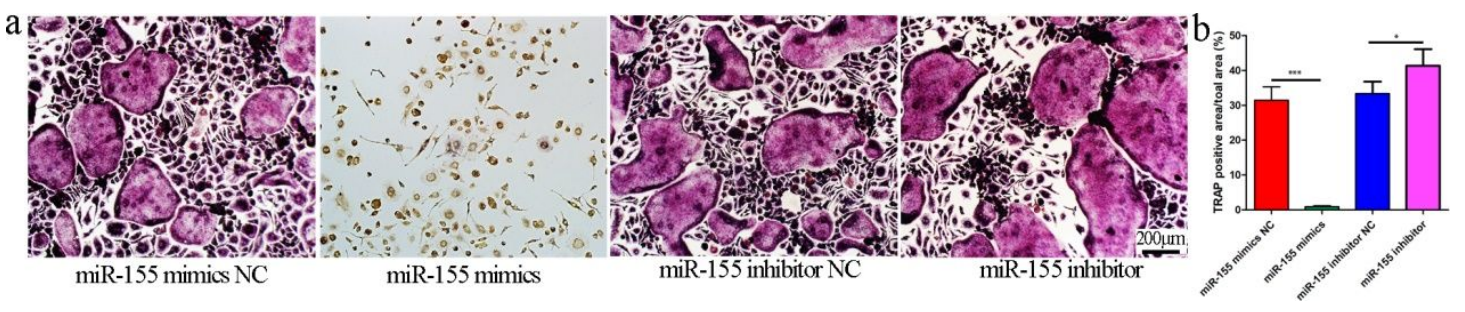

Figure S9. The effect of miR-155 mimic and inhibitor on BMM differentiation. (a) Representative images of TRAP-positive cells in the miR-155 mimic negative control group, miR-155 mimic group, miR-155 inhibitor negative control group and miR-155 inhibitor group (scale bar $200 \mu \mathrm{m}$ ). (b) Quantitative analysis of the area of TRAP-positive osteoclasts. Data are presented as the mean $\pm \mathrm{SD},\left({ }^{*} p<0.05, * * * p<0.001\right)$.

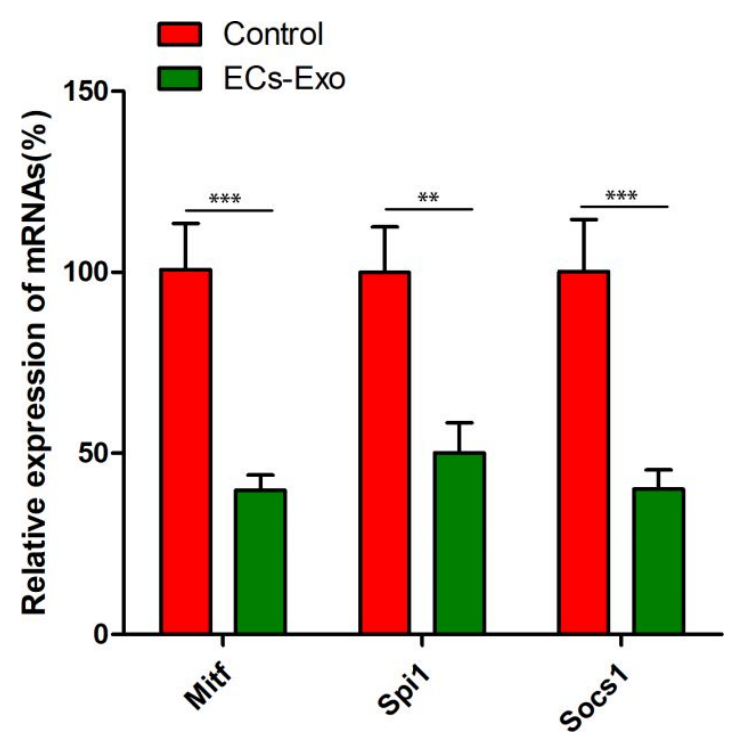

Figure S10. The mRNA expression of Mitf, Spi1, and Socs1 in EC-Exos-treated BMMs. Data are presented as the mean $\pm \mathrm{SD},(* * p<0.01, * * * p<0.001)$. 

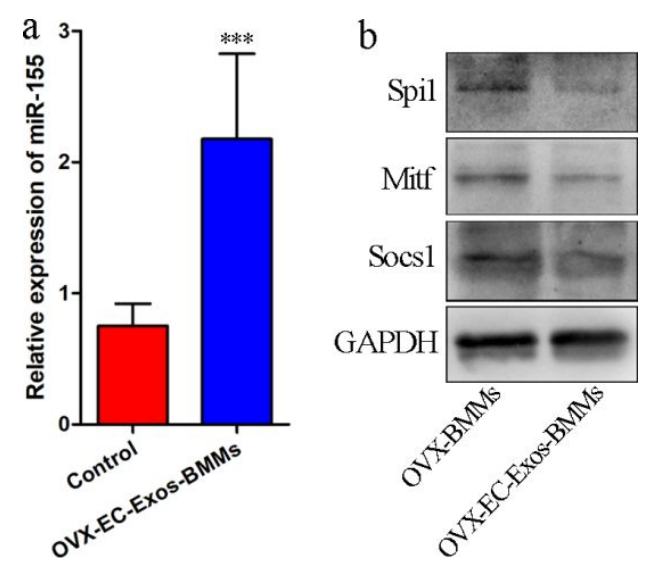

Figure S11. EC-Exos transferred miR-155 to BMMs in vivo. (a) The mRNA expression of miR-155 in BMMs isolated from EC-Exos-treated mice and control mice $(* * * p<0.001)$. Data are presented as the mean \pm SD. (b) The expression of Spi1, Mitf, and Socs1 in BMMs isolated from EC-Exos-treated mice were inhibited compared with that of control mice.

\section{Reference}

(1) Chen, X.; Zhi, X.; Pan, P.; Cui, J.; Cao, L.; Weng, W.; Zhou, Q.; Wang, L.; Zhai, X.; Zhao, Q.; Hu, H.; Huang, B.; Su, J., Matrine Prevents Bone Loss in Ovariectomized Mice by Inhibiting Rankl-Induced Osteoclastogenesis. FASEB J. 2017, 31, 4855-4865.

(2) Chen, X.; Zhi, X.; Wang, J.; Su, J., Rankl Signaling in Bone Marrow Mesenchymal Stem Cells Negatively Regulates Osteoblastic Bone Formation. Bone Res. 2018, 6, 34 


\section{Table S1. Highly expressed proteins in EC-Exos compared with BMSC-Exos.}

\begin{tabular}{|c|c|c|c|}
\hline Accession & Significance & $\begin{array}{l}\text { Group } \\
\text { (Ratio) }\end{array}$ & Description \\
\hline P01023 & 55.79 & $12.79: 1.00$ & $\begin{array}{l}\text { Alpha-2-macroglobulin OS=Homo sapiens } \mathrm{OX}=9606 \mathrm{GN}=\mathrm{A} 2 \mathrm{M} P E=1 \\
\mathrm{SV}=3\end{array}$ \\
\hline P07996 & 49.11 & $3.31: 1.00$ & $\begin{array}{l}\text { Thrombospondin-1 OS=Homo sapiens OX=9606 GN=THBS1 PE=1 } \\
\text { SV=2 }\end{array}$ \\
\hline P04264 & 48.14 & $1.78: 1.00$ & $\begin{array}{l}\text { Keratin type II cytoskeletal } 1 \text { OS=Homo sapiens OX=9606 } \\
\text { GN=KRT1 PE=1 SV=6 }\end{array}$ \\
\hline P35527 & 38.22 & $2.01: 1.00$ & $\begin{array}{l}\text { Keratin type I cytoskeletal } 9 \text { OS=Homo sapiens OX=9606 } \\
\text { GN=KRT9 PE=1 SV=3 }\end{array}$ \\
\hline $\begin{array}{l}\text { A0A087 } \\
\text { WTA8 }\end{array}$ & 34.49 & $2.97: 1.00$ & $\begin{array}{l}\text { Collagen alpha-2(I) chain OS=Homo sapiens } \mathrm{OX}=9606 \mathrm{GN}=\mathrm{COL} 1 \mathrm{~A} 2 \\
\mathrm{PE}=1 \mathrm{SV}=1\end{array}$ \\
\hline P08123 & 34.49 & $2.97: 1.00$ & $\begin{array}{l}\text { Collagen alpha-2(I) chain OS=Homo sapiens } 0 X=9606 \text { GN=COL1A2 } \\
P E=1 S V=7\end{array}$ \\
\hline P35908 & 34.2 & $1.98: 1.00$ & $\begin{array}{l}\text { Keratin type II cytoskeletal } 2 \text { epidermal OS=Homo sapiens } \\
\text { OX=9606 GN=KRT2 PE=1 SV=2 }\end{array}$ \\
\hline P13645 & 33.18 & $1.86: 1.00$ & $\begin{array}{l}\text { Keratin type I cytoskeletal } 10 \text { OS=Homo sapiens OX=9606 } \\
\text { GN=KRT10 PE=1 SV=6 }\end{array}$ \\
\hline P09486 & 29.94 & $1.67: 1.00$ & SPARC OS=Homo sapiens OX=9606 GN=SPARC PE=1 SV=1 \\
\hline $\begin{array}{l}\text { A0A140 } \\
\text { TA32 }\end{array}$ & 29.23 & $1.95: 1.00$ & Complement C4-A OS=Homo sapiens OX=9606 GN=C4A PE=1 SV=1 \\
\hline $\begin{array}{l}\text { A0A140 } \\
\text { TA49 }\end{array}$ & 29.23 & $1.95: 1.00$ & Complement C4-A OS=Homo sapiens OX=9606 GN=C4A PE=1 SV=1 \\
\hline $\begin{array}{l}\text { A0A140 } \\
\text { TA44 }\end{array}$ & 29.23 & $1.95: 1.00$ & Complement C4-A OS=Homo sapiens OX=9606 GN=C4A PE=1 SV=1 \\
\hline $\begin{array}{l}\text { AOA0G2 } \\
\text { JPRO }\end{array}$ & 29.23 & $1.95: 1.00$ & Complement C4-A OS=Homo sapiens OX=9606 GN=C4A PE=1 SV=1 \\
\hline P0COL4 & 29.23 & $1.95: 1.00$ & Complement C4-A OS=Homo sapiens OX=9606 GN=C4A PE=1 SV=2 \\
\hline $\begin{array}{l}\text { A0A1B0 } \\
\text { GU03 }\end{array}$ & 24.09 & $2.12: 1.00$ & Uncharacterized protein OS=Homo sapiens OX=9606 PE=1 SV=1 \\
\hline $\begin{array}{l}\text { A0A1B0 } \\
\text { GV23 }\end{array}$ & 24.09 & 2.12:1.00 & Cathepsin D OS=Homo sapiens OX=9606 GN=CTSD PE=1 SV=1 \\
\hline $\begin{array}{l}\text { A0A1B0 } \\
\text { GW44 }\end{array}$ & 24.09 & 2.12:1.00 & Cathepsin D OS=Homo sapiens OX=9606 GN=CTSD PE=1 SV=1 \\
\hline $\begin{array}{l}\text { A0A1B0 } \\
\text { GVD5 }\end{array}$ & 24.09 & 2.12:1.00 & Cathepsin D OS=Homo sapiens OX=9606 GN=CTSD PE=1 SV=1 \\
\hline $\begin{array}{l}\text { A0A1B0 } \\
\text { GWE8 }\end{array}$ & 24.09 & 2.12:1.00 & Cathepsin D OS=Homo sapiens OX=9606 GN=CTSD PE=1 SV=1 \\
\hline
\end{tabular}




\begin{tabular}{|c|c|c|c|}
\hline P07339 & 24.09 & 2.12:1.00 & Cathepsin D OS=Homo sapiens OX=9606 GN=CTSD PE=1 SV=1 \\
\hline $\begin{array}{l}\text { A0A1B0 } \\
\text { GU92 }\end{array}$ & 24.09 & 2.12:1.00 & Uncharacterized protein OS=Homo sapiens $\mathrm{OX}=9606 \mathrm{PE}=1 \mathrm{SV}=1$ \\
\hline H7C469 & 24.09 & 2.12:1.00 & $\begin{array}{l}\text { Uncharacterized protein (Fragment) OS=Homo sapiens OX=9606 } \\
\mathrm{PE}=1 \mathrm{SV}=7\end{array}$ \\
\hline H7C1V0 & 24.09 & 2.12:1.00 & $\begin{array}{l}\text { Cathepsin D (Fragment) OS=Homo sapiens OX=9606 GN=CTSD } \\
\mathrm{PE}=1 \mathrm{SV}=1\end{array}$ \\
\hline $\begin{array}{l}\text { A0A0A0 } \\
\text { MSA4 }\end{array}$ & 22.99 & 2.16:1.00 & $\begin{array}{l}\text { Band 4.1-like protein } 3 \text { OS=Homo sapiens OX=9606 GN=EPB41L3 } \\
\mathrm{PE}=1 \mathrm{SV}=1\end{array}$ \\
\hline Q9Y490 & 22.96 & $1.34: 1.00$ & Talin-1 OS=Homo sapiens OX=9606 GN=TLN1 PE=1 SV=3 \\
\hline P04003 & 22.18 & $1.30: 1.00$ & $\begin{array}{l}\text { C4b-binding protein alpha chain OS=Homo sapiens OX=9606 } \\
\text { GN=C4BPA PE }=1 \mathrm{SV}=2\end{array}$ \\
\hline P05556 & 20.05 & $1.29: 1.00$ & Integrin beta- 1 OS=Homo sapiens $\mathrm{OX}=9606 \mathrm{GN}=\mathrm{ITGB} 1 \mathrm{PE}=1 \mathrm{SV}=2$ \\
\hline P32119 & 19.3 & $1.14: 1.00$ & Peroxiredoxin-2 OS=Homo sapiens OX=9606 GN=PRDX2 PE=1 SV=5 \\
\hline A6NIW5 & 19.3 & 1.14:1.00 & $\begin{array}{l}\text { Peroxiredoxin } 2 \text { isoform CRA_a OS=Homo sapiens OX=9606 } \\
\text { GN=PRDX2 PE=1 SV=2 }\end{array}$ \\
\hline P11717 & 19.25 & 1.51:1.00 & $\begin{array}{l}\text { Cation-independent mannose-6-phosphate receptor OS=Homo } \\
\text { sapiens } 0 X=9606 \mathrm{GN}=\mathrm{IGF} 2 \mathrm{R} \mathrm{PE}=1 \mathrm{SV}=3\end{array}$ \\
\hline P00734 & 18.51 & 1.18:1.00 & Prothrombin OS=Homo sapiens $\mathrm{OX}=9606 \mathrm{GN}=\mathrm{F} 2 \mathrm{PE}=1 \mathrm{SV}=2$ \\
\hline E9PIT3 & 18.51 & 1.18:1.00 & Prothrombin OS=Homo sapiens OX=9606 GN=F2 PE=1 SV=1 \\
\hline P20742 & 17.44 & 15.07:1.00 & $\begin{array}{l}\text { Pregnancy zone protein } \mathrm{OS}=\text { Homo sapiens } \mathrm{OX}=9606 \mathrm{GN}=\mathrm{PZP} P E=1 \\
\mathrm{SV}=4\end{array}$ \\
\hline J3QSU6 & 16.93 & $27.27: 1.00$ & Tenascin OS=Homo sapiens $\mathrm{OX}=9606 \mathrm{GN}=\mathrm{TNC} \mathrm{PE}=1 \mathrm{SV}=1$ \\
\hline P24821 & 16.93 & 27.27:1.00 & Tenascin OS=Homo sapiens $\mathrm{OX}=9606 \mathrm{GN}=\mathrm{TNC} \mathrm{PE}=1 \mathrm{SV}=3$ \\
\hline F5H7V9 & 16.93 & $27.27: 1.00$ & Tenascin OS=Homo sapiens $\mathrm{OX}=9606 \mathrm{GN}=\mathrm{TNC} \mathrm{PE}=1 \mathrm{SV}=1$ \\
\hline P13647 & 15.94 & 1.28:1.00 & $\begin{array}{l}\text { Keratin type II cytoskeletal } 5 \text { OS=Homo sapiens OX=9606 } \\
\text { GN=KRT5 PE=1 SV=3 }\end{array}$ \\
\hline P68366 & 15.29 & 1.83:1.00 & $\begin{array}{l}\text { Tubulin alpha-4A chain OS=Homo sapiens OX=9606 GN=TUBA4A } \\
\mathrm{PE}=1 \mathrm{SV}=1\end{array}$ \\
\hline P02533 & 14.48 & 1.31:1.00 & $\begin{array}{l}\text { Keratin type I cytoskeletal } 14 \text { OS=Homo sapiens OX=9606 } \\
\text { GN=KRT14 PE=1 SV=4 }\end{array}$ \\
\hline P02461 & 13.64 & $1.13: 1.00$ & $\begin{array}{l}\text { Collagen alpha-1(III) chain OS=Homo sapiens OX=9606 GN=COL3A1 } \\
\mathrm{PE}=1 \mathrm{SV}=4\end{array}$ \\
\hline P15169 & 13 & 1.12:1.00 & $\begin{array}{l}\text { Carboxypeptidase } \mathrm{N} \text { catalytic chain } \mathrm{OS}=\text { Homo sapiens } \mathrm{OX}=9606 \\
\mathrm{GN}=\mathrm{CPN} 1 \mathrm{PE}=1 \mathrm{SV}=1\end{array}$ \\
\hline B1AP58 & 13 & 1.12:1.00 & $\begin{array}{l}\text { Carboxypeptidase } \mathrm{N} \text { catalytic chain (Fragment) OS=Homo sapiens } \\
\text { OX=9606 GN=CPN1 PE=1 SV=1 }\end{array}$ \\
\hline
\end{tabular}




\section{Table S2. Highly expressed proteins in EC-Exos compared with MC3T3-Exos.}

\begin{tabular}{|c|c|c|c|}
\hline Accession & Significance & $\begin{array}{l}\text { Group Profile } \\
\text { (Ratio) }\end{array}$ & Description \\
\hline P04264 & 31.45 & 1.00:0.81 & Keratin type II cytoskeletal 1 OS=Homo sapiens OX=9606 GN=KRT1 PE=1 SV=6 \\
\hline P09486 & 30.62 & $1.00: 0.63$ & SPARC OS=Homo sapiens OX=9606 GN=SPARC PE=1 SV=1 \\
\hline BOYJC4 & 29.75 & 1.00:0.71 & Vimentin variant 3 OS=Homo sapiens OX=9606 GN=VIM PE=1 SV=1 \\
\hline P08670 & 29.75 & 1.00:0.71 & Vimentin OS=Homo sapiens OX=9606 GN=VIM PE=1 SV=4 \\
\hline P05543 & 28.64 & $1.00: 0.74$ & Thyroxine-binding globulin OS=Homo sapiens OX=9606 GN=SERPINA7 PE=1 SV=2 \\
\hline P08697 & 27.85 & $1.00: 0.34$ & Alpha-2-antiplasmin OS=Homo sapiens OX=9606 GN=SERPINF2 PE=1 SV=3 \\
\hline $\begin{array}{l}\text { A0A0G2 } \\
\text { JPA8 }\end{array}$ & 27.85 & $1.00: 0.34$ & Alpha-2-antiplasmin OS=Homo sapiens OX=9606 GN=SERPINF2 PE=1 SV=1 \\
\hline C9JMH6 & 27.85 & $1.00: 0.34$ & $\begin{array}{l}\text { Alpha-2-antiplasmin (Fragment) OS=Homo sapiens OX=9606 GN=SERPINF2 PE=1 } \\
\text { SV=1 }\end{array}$ \\
\hline $\begin{array}{l}\text { A0A0J9 } \\
\text { YY65 }\end{array}$ & 27.85 & 1.00:0.34 & $\begin{array}{l}\text { Alpha-2-antiplasmin (Fragment) OS=Homo sapiens OX=9606 GN=SERPINF2 PE=1 } \\
\text { SV=1 }\end{array}$ \\
\hline Q9BS19 & 27.28 & 1.00:0.64 & HPX protein OS=Homo sapiens OX=9606 GN=HPX PE=1 SV=1 \\
\hline P02790 & 27.28 & $1.00: 0.64$ & Hemopexin OS=Homo sapiens OX=9606 GN=HPX PE=1 SV=2 \\
\hline P05556 & 27.08 & $1.00: 0.46$ & Integrin beta- 1 OS=Homo sapiens OX=9606 GN=ITGB1 PE=1 SV=2 \\
\hline P23526 & 25.54 & 1.00:0.70 & Adenosylhomocysteinase OS=Homo sapiens OX=9606 GN=AHCY PE=1 SV=4 \\
\hline P35527 & 24.96 & 1.00:0.58 & Keratin type I cytoskeletal 9 OS=Homo sapiens OX=9606 GN=KRT9 PE=1 SV=3 \\
\hline P00734 & 24.26 & 1.00:0.79 & Prothrombin OS=Homo sapiens OX=9606 GN=F2 PE=1 SV=2 \\
\hline E9PIT3 & 24.26 & 1.00:0.79 & Prothrombin OS=Homo sapiens OX=9606 GN=F2 PE=1 SV=1 \\
\hline E7EUT5 & 22.97 & 1.00:0.81 & $\begin{array}{l}\text { Glyceraldehyde-3-phosphate dehydrogenase OS=Homo sapiens OX=9606 } \\
\text { GN=GAPDH PE=1 SV=1 }\end{array}$ \\
\hline P04406 & 22.97 & 1.00:0.81 & $\begin{array}{l}\text { Glyceraldehyde-3-phosphate dehydrogenase OS=Homo sapiens OX=9606 } \\
\text { GN=GAPDH PE=1 SV=3 }\end{array}$ \\
\hline 095445 & 22.45 & 1.00:0.71 & Apolipoprotein M OS=Homo sapiens OX=9606 GN=APOM PE=1 SV=2 \\
\hline Q5SRP5 & 22.45 & 1.00:0.71 & Apolipoprotein M OS=Homo sapiens OX=9606 GN=APOM PE=1 SV=1 \\
\hline P05546 & 22.21 & $1.00: 0.63$ & Heparin cofactor 2 OS=Homo sapiens OX=9606 GN=SERPIND1 PE=1 SV=3 \\
\hline P62805 & 21.27 & 1.00:0.76 & Histone H4 OS=Homo sapiens OX=9606 GN=HIST1H4A PE=1 SV=2 \\
\hline Q5VTE0 & 20.81 & 1.00:0.85 & $\begin{array}{l}\text { Putative elongation factor 1-alpha-like } 3 \text { OS=Homo sapiens OX=9606 } \\
\text { GN=EEF1A1P5 } P E=5 \mathrm{SV}=1\end{array}$ \\
\hline P68104 & 20.81 & 1.00:0.85 & Elongation factor 1 -alpha 1 OS=Homo sapiens OX=9606 GN=EEF1A1 PE=1 SV=1 \\
\hline Q15582 & 20.55 & 1.00:0.70 & $\begin{array}{l}\text { Transforming growth factor-beta-induced protein ig-h3 OS=Homo sapiens } \\
\text { OX=9606 GN=TGFBI PE=1 SV=1 }\end{array}$ \\
\hline $\begin{array}{l}\text { AOAOAO } \\
\text { MSA4 }\end{array}$ & 19.88 & 1.00:0.49 & Band 4.1-like protein 3 OS=Homo sapiens OX=9606 GN=EPB41L3 PE=1 SV=1 \\
\hline HOY3H2 & 19.11 & 1.00:0.32 & ATP-binding cassette sub-family A member 3 OS=Homo sapiens OX=9606 \\
\hline
\end{tabular}




\begin{tabular}{|c|c|c|c|}
\hline & & & $\mathrm{GN}=\mathrm{ABCA} 3 \mathrm{PE}=1 \mathrm{SV}=1$ \\
\hline Q99758 & 19.11 & 1.00:0.32 & $\begin{array}{l}\text { ATP-binding cassette sub-family } A \text { member } 3 \text { OS=Homo sapiens } O X=9606 \\
G N=A B C A 3 P E=1 \mathrm{SV}=2\end{array}$ \\
\hline P14543 & 18.78 & $1.00: 0.40$ & Nidogen-1 OS=Homo sapiens OX=9606 GN=NID1 PE=1 SV=3 \\
\hline 095497 & 17.64 & $1.00: 0.73$ & Pantetheinase OS=Homo sapiens OX=9606 GN=VNN1 PE=1 SV=2 \\
\hline Q9NTU7 & 16.71 & 1.00:0.79 & Cerebellin-4 OS=Homo sapiens OX=9606 GN=CBLN4 PE=1 SV=1 \\
\hline $\begin{array}{l}\text { A0A087 } \\
\text { W } \\
\text { WY3 }\end{array}$ & 15.89 & 1.00:0.87 & Filamin-A OS=Homo sapiens OX=9606 GN=FLNA PE=1 SV=1 \\
\hline Q60FE5 & 15.89 & 1.00:0.87 & Filamin-A OS=Homo sapiens OX=9606 GN=FLNA PE=1 SV=1 \\
\hline P21333 & 15.89 & 1.00:0.87 & Filamin-A OS=Homo sapiens OX=9606 GN=FLNA PE=1 SV=4 \\
\hline P20742 & 15.05 & $1.00: 0.33$ & Pregnancy zone protein OS=Homo sapiens OX=9606 GN=PZP PE=1 SV=4 \\
\hline P26038 & 13.57 & 1.00:0.65 & Moesin OS=Homo sapiens OX=9606 GN=MSN PE=1 SV=3 \\
\hline Q96PD5 & 13.32 & 1.00:0.67 & $\begin{array}{l}\text { N-acetylmuramoyl-L-alanine amidase OS=Homo sapiens OX=9606 GN=PGLYRP2 } \\
P E=1 \mathrm{SV}=1\end{array}$ \\
\hline P62258 & 13.2 & $1.00: 0.80$ & 14-3-3 protein epsilon OS=Homo sapiens OX=9606 GN=YWHAE PE=1 SV=1 \\
\hline P02649 & 13.14 & 1.00:0.91 & Apolipoprotein E OS=Homo sapiens OX=9606 GN=APOE PE=1 SV=1 \\
\hline
\end{tabular}


Table S3. Differentially expressed miRNAs in EC-Exos-treated BMMs compared with control BMMs.

\begin{tabular}{|c|c|c|c|c|}
\hline miRNA ID & $\begin{array}{l}\log 2 \text { Ratio } \\
\text { (EC-/Control) }\end{array}$ & $\begin{array}{ll}\text { Up down } & \text { degulation }\end{array}$ & $P$ value & $Q$ value \\
\hline mmu-miR-142a-3p & 2.429712 & UP & 0 & 0 \\
\hline mmu-miR-155-5p & 4.677136 & UP & 0 & 0 \\
\hline mmu-miR-378a-3p & -1.03373 & DOWN & 0 & 0 \\
\hline mmu-miR-532-5p & -1.55502 & DOWN & 0 & 0 \\
\hline mmu-let-7c-5p & -1.04679 & DOWN & 0 & 0 \\
\hline mmu-miR-5099 & -2.01183 & DOWN & 0 & 0 \\
\hline mmu-miR-15a-5p & 1.254114 & UP & 0 & 0 \\
\hline mmu-miR-29b-3p & 2.509361 & UP & 0 & 0 \\
\hline mmu-miR-30d-5p & 1.155576 & UP & 0 & 0 \\
\hline mmu-miR-30b-5p & 1.194156 & UP & 0 & 0 \\
\hline mmu-miR-142a-5p & 1.403926 & UP & 0 & 0 \\
\hline mmu-miR-98-5p & -1.07973 & DOWN & 0 & 0 \\
\hline mmu-miR-500-3p & 1.666049 & UP & 0 & 0 \\
\hline mmu-miR-149-5p & -2.5354 & DOWN & 0 & 0 \\
\hline mmu-miR-1198-5p & -1.04678 & DOWN & 0 & 0 \\
\hline mmu-miR-125a-5p & 1.887339 & UP & 0 & 0 \\
\hline mmu-miR-700-3p & -3.31579 & DOWN & 0 & 0 \\
\hline mmu-miR-351-5p & -2.00738 & DOWN & 0 & 0 \\
\hline mmu-miR-674-5p & 1.98059 & UP & 0 & 0 \\
\hline mmu-miR-10a-5p & -1.0058 & DOWN & 0 & 0 \\
\hline mmu-miR-142b & 2.821036 & UP & 0 & 0 \\
\hline mmu-miR-106b-5p & 2.217663 & UP & 0 & 0 \\
\hline mmu-let-7c-2-3p & -4.77232 & DOWN & 0 & 0 \\
\hline mmu-miR-17-5p & 1.09017 & UP & 0 & 0 \\
\hline mmu-miR-30c-5p & 1.765881 & UP & 0 & 0 \\
\hline mmu-miR-574-3p & -1.39839 & DOWN & 0 & 0 \\
\hline mmu-miR-140-3p & 1.323911 & UP & 0 & 0 \\
\hline mmu-miR-532-3p & -1.52366 & DOWN & 0 & 0 \\
\hline mmu-miR-125b-5p & 1.25825 & UP & 0 & 0 \\
\hline mmu-miR-191-3p & -2.47811 & DOWN & 0 & 0 \\
\hline mmu-miR-30e-5p & 2.243999 & UP & 0 & 0 \\
\hline mmu-miR-186-5p & 1.66033 & UP & 0 & 0 \\
\hline mmu-miR-340-3p & -1.08603 & DOWN & 0 & 0 \\
\hline mmu-miR-1983 & -1.31819 & DOWN & 0 & 0 \\
\hline
\end{tabular}




\begin{tabular}{|c|c|c|c|c|}
\hline mmu-miR-1981-5p & -1.14712 & DOWN & 0 & 0 \\
\hline mmu-miR-101a-3p & 2.631633 & UP & 0 & 0 \\
\hline mmu-miR-3068-5p & -1.11682 & DOWN & 0 & 0 \\
\hline mmu-miR-3074-2-3p & 1.236523 & UP & 0 & 0 \\
\hline mmu-miR-130b-5p & -2.01661 & DOWN & 0 & 0 \\
\hline mmu-miR-151-3p & 1.177819 & UP & 0 & 0 \\
\hline mmu-miR-32-5p & 1.259576 & UP & 0 & 0 \\
\hline mmu-miR-19a-3p & 2.369599 & UP & 0 & 0 \\
\hline mmu-miR-27a-5p & -1.01414 & DOWN & 0 & 0 \\
\hline mmu-miR-138-5p & -1.4153 & DOWN & 0 & 0 \\
\hline mmu-miR-23b-5p & -1.54079 & DOWN & 0 & 0 \\
\hline mmu-miR-511-3p & -1.00313 & DOWN & 0 & 0 \\
\hline mmu-miR-326-3p & 1.096269 & UP & 0 & 0 \\
\hline mmu-let-7i-3p & 1.117927 & UP & 0 & 0 \\
\hline mmu-miR-425-3p & -1.6046 & DOWN & 0 & 0 \\
\hline mmu-miR-3065-5p & 3.640987 & UP & 0 & 0 \\
\hline mmu-miR-362-3p & 2.226944 & UP & 0 & 0 \\
\hline mmu-miR-339-5p & 1.249561 & UP & 0 & 0 \\
\hline mmu-miR-511-5p & -1.81288 & DOWN & 0 & 0 \\
\hline mmu-miR-27b-5p & -1.45267 & DOWN & 0 & 0 \\
\hline mmu-miR-188-5p & 1.389234 & UP & 0 & 0 \\
\hline mmu-miR-872-3p & -1.26963 & DOWN & 0 & 0 \\
\hline mmu-miR-542-5p & -1.03691 & DOWN & 0 & 0 \\
\hline mmu-miR-503-5p & 1.158283 & UP & 0 & 0 \\
\hline mmu-miR-147-3p & 5.484663 & UP & 0 & 0 \\
\hline novel_mir3 & 2.99128 & UP & 0 & 0 \\
\hline mmu-miR-674-3p & 1.668517 & UP & 0 & 0 \\
\hline mmu-miR-100-5p & 1.052188 & UP & 0 & 0 \\
\hline mmu-miR-744-3p & -2.38093 & DOWN & 0 & 0 \\
\hline mmu-miR-122-5p & -2.73172 & DOWN & 0 & 0 \\
\hline mmu-miR-130b-3p & 1.428345 & UP & 0 & 0 \\
\hline mmu-miR-130a-3p & 2.312796 & UP & $2.12 \mathrm{E}-303$ & $6.49 \mathrm{E}-303$ \\
\hline mmu-miR-467h & -3.08967 & DOWN & $1.09 \mathrm{E}-247$ & $3.22 \mathrm{E}-247$ \\
\hline mmu-miR-298-5p & -2.0429 & DOWN & $1.07 \mathrm{E}-227$ & $3.10 \mathrm{E}-227$ \\
\hline mmu-let-7f-1-3p & -2.00531 & DOWN & 4.43E-218 & $1.26 \mathrm{E}-217$ \\
\hline novel_mir243 & -1.18573 & DOWN & $1.18 \mathrm{E}-208$ & $3.23 \mathrm{E}-208$ \\
\hline mmu-miR-30a-5p & 1.420409 & UP & $5.79 \mathrm{E}-202$ & $1.57 \mathrm{E}-201$ \\
\hline mmu-miR-466d-3p & 1.924765 & UP & $8.13 \mathrm{E}-182$ & $2.16 \mathrm{E}-181$ \\
\hline
\end{tabular}




\begin{tabular}{|c|c|c|c|c|}
\hline mmu-miR-210-3p & 1.693969 & UP & $3.28 \mathrm{E}-175$ & $8.62 \mathrm{E}-175$ \\
\hline mmu-miR-199b-3p & 1.015137 & UP & $4.46 \mathrm{E}-174$ & $1.17 \mathrm{E}-173$ \\
\hline mmu-miR-331-3p & 1.604304 & UP & $2.22 \mathrm{E}-171$ & $5.71 \mathrm{E}-171$ \\
\hline mmu-miR-29a-5p & 1.337248 & UP & $3.34 \mathrm{E}-170$ & $8.55 \mathrm{E}-170$ \\
\hline mmu-miR-141-3p & 2.006964 & UP & $1.28 \mathrm{E}-168$ & $3.26 \mathrm{E}-168$ \\
\hline mmu-miR-24-1-5p & 1.265188 & UP & $8.81 \mathrm{E}-164$ & $2.22 \mathrm{E}-163$ \\
\hline mmu-miR-669a-3p & 1.988635 & UP & $5.80 \mathrm{E}-162$ & $1.45 \mathrm{E}-161$ \\
\hline mmu-miR-450b-3p & -1.3165 & DOWN & $2.75 \mathrm{E}-156$ & $6.84 \mathrm{E}-156$ \\
\hline mmu-miR-139-3p & -1.05927 & DOWN & $1.40 \mathrm{E}-153$ & $3.46 \mathrm{E}-153$ \\
\hline mmu-miR-5126 & 2.703134 & UP & $1.34 \mathrm{E}-139$ & $3.24 \mathrm{E}-139$ \\
\hline mmu-miR-652-5p & -1.21609 & DOWN & $3.16 \mathrm{E}-139$ & $7.56 \mathrm{E}-139$ \\
\hline mmu-miR-31-5p & 1.381173 & UP & $9.41 \mathrm{E}-139$ & $2.24 \mathrm{E}-138$ \\
\hline mmu-miR-126a-5p & 1.684444 & UP & $2.78 \mathrm{E}-138$ & $6.56 \mathrm{E}-138$ \\
\hline mmu-miR-193a-3p & 1.72466 & UP & 7.34E-131 & $1.70 \mathrm{E}-130$ \\
\hline mmu-miR-338-3p & 1.146347 & UP & $1.20 \mathrm{E}-121$ & $2.76 \mathrm{E}-121$ \\
\hline novel_mir218 & 1.95899 & UP & $6.76 \mathrm{E}-112$ & $1.52 \mathrm{E}-111$ \\
\hline mmu-miR-214-3p & 1.77763 & UP & $8.39 \mathrm{E}-110$ & $1.87 \mathrm{E}-109$ \\
\hline mmu-miR-466f-3p & 2.552517 & UP & $1.41 \mathrm{E}-106$ & $3.12 \mathrm{E}-106$ \\
\hline novel_mir307 & 2.858076 & UP & $2.84 \mathrm{E}-102$ & $6.23 \mathrm{E}-102$ \\
\hline mmu-miR-669o-3p & 2.175791 & UP & $2.27 \mathrm{E}-100$ & $4.95 \mathrm{E}-100$ \\
\hline mmu-miR-34a-3p & 2.013965 & UP & $2.95 \mathrm{E}-91$ & $6.33 \mathrm{E}-91$ \\
\hline mmu-miR-7062-5p & -1.08916 & DOWN & $2.07 \mathrm{E}-89$ & $4.42 \mathrm{E}-89$ \\
\hline mmu-miR-467a-3p & 4.130917 & UP & $2.86 \mathrm{E}-89$ & $6.06 \mathrm{E}-89$ \\
\hline mmu-miR-203-3p & 1.21501 & UP & $4.87 \mathrm{E}-88$ & $1.03 \mathrm{E}-87$ \\
\hline mmu-miR-145a-5p & 2.021489 & UP & $1.64 \mathrm{E}-87$ & $3.44 \mathrm{E}-87$ \\
\hline mmu-miR-296-3p & -1.37807 & DOWN & $4.42 \mathrm{E}-86$ & $9.20 \mathrm{E}-86$ \\
\hline mmu-miR-486b-5p & -1.13082 & DOWN & $5.23 \mathrm{E}-83$ & $1.06 \mathrm{E}-82$ \\
\hline mmu-miR-148b-5p & 2.718999 & UP & $1.94 \mathrm{E}-80$ & $3.91 \mathrm{E}-80$ \\
\hline mmu-miR-1306-3p & -1.03749 & DOWN & $1.57 \mathrm{E}-78$ & $3.13 \mathrm{E}-78$ \\
\hline mmu-miR-7091-3p & -1.72791 & DOWN & $9.19 \mathrm{E}-77$ & $1.81 \mathrm{E}-76$ \\
\hline novel_mir75 & 3.667185 & UP & $2.57 \mathrm{E}-76$ & $5.04 \mathrm{E}-76$ \\
\hline mmu-miR-30c-1-3p & -1.60379 & DOWN & $8.01 \mathrm{E}-76$ & $1.56 \mathrm{E}-75$ \\
\hline mmu-miR-466b-3p & 1.484661 & UP & $1.38 \mathrm{E}-74$ & $2.68 \mathrm{E}-74$ \\
\hline mmu-miR-1934-5p & -1.54454 & DOWN & $3.17 \mathrm{E}-71$ & $6.10 \mathrm{E}-71$ \\
\hline mmu-miR-32-3p & -1.1794 & DOWN & $1.47 \mathrm{E}-69$ & $2.80 \mathrm{E}-69$ \\
\hline mmu-miR-101c & 3.484298 & UP & $9.02 \mathrm{E}-69$ & $1.71 \mathrm{E}-68$ \\
\hline mmu-miR-760-3p & -1.04711 & DOWN & $4.36 \mathrm{E}-65$ & $8.01 \mathrm{E}-65$ \\
\hline mmu-miR-501-5p & 1.924206 & UP & $5.39 \mathrm{E}-64$ & $9.81 \mathrm{E}-64$ \\
\hline
\end{tabular}




\begin{tabular}{|c|c|c|c|c|}
\hline mmu-miR-7669-3p & -1.68178 & DOWN & $3.85 \mathrm{E}-62$ & $6.90 \mathrm{E}-62$ \\
\hline mmu-miR-365-2-5p & -2.51122 & DOWN & $2.76 \mathrm{E}-61$ & 4.91E-61 \\
\hline mmu-miR-466c-3p & 1.646587 & UP & $3.00 \mathrm{E}-61$ & $5.30 \mathrm{E}-61$ \\
\hline mmu-miR-361-3p & 2.37686 & UP & $3.20 \mathrm{E}-58$ & $5.61 \mathrm{E}-58$ \\
\hline mmu-miR-16-2-3p & 1.684418 & UP & $6.95 \mathrm{E}-58$ & $1.20 \mathrm{E}-57$ \\
\hline novel_mir47 & 1.643123 & UP & $1.17 \mathrm{E}-56$ & $2.02 \mathrm{E}-56$ \\
\hline mmu-miR-103-2-5p & 2.254065 & UP & $1.30 \mathrm{E}-54$ & $2.24 \mathrm{E}-54$ \\
\hline novel_mir217 & 3.419777 & UP & $4.45 \mathrm{E}-54$ & $7.60 \mathrm{E}-54$ \\
\hline novel_mir94 & 3.283564 & UP & $1.57 \mathrm{E}-52$ & $2.67 \mathrm{E}-52$ \\
\hline mmu-miR-3061-5p & -1.37166 & DOWN & $3.10 \mathrm{E}-51$ & $5.23 \mathrm{E}-51$ \\
\hline mmu-miR-5627-5p & -1.46193 & DOWN & $1.72 \mathrm{E}-49$ & $2.89 \mathrm{E}-49$ \\
\hline mmu-miR-146a-3p & 3.090275 & UP & 4.49E-49 & $7.48 \mathrm{E}-49$ \\
\hline mmu-miR-219a-5p & 2.960363 & UP & 4.94E-49 & $8.18 \mathrm{E}-49$ \\
\hline mmu-miR-144-3p & 3.136966 & UP & $1.48 \mathrm{E}-48$ & $2.43 \mathrm{E}-48$ \\
\hline mmu-miR-365-3p & 2.211917 & UP & $1.92 \mathrm{E}-48$ & $3.16 \mathrm{E}-48$ \\
\hline mmu-miR-1947-5p & -1.15629 & DOWN & $1.39 \mathrm{E}-47$ & $2.26 \mathrm{E}-47$ \\
\hline mmu-miR-187-3p & -1.57045 & DOWN & $6.55 \mathrm{E}-47$ & $1.05 \mathrm{E}-46$ \\
\hline novel_mir305 & 1.728906 & UP & $7.07 \mathrm{E}-45$ & $1.12 \mathrm{E}-44$ \\
\hline novel_mir215 & -2.96312 & DOWN & $1.01 \mathrm{E}-43$ & $1.59 \mathrm{E}-43$ \\
\hline mmu-miR-1930-5p & -1.03663 & DOWN & $1.23 \mathrm{E}-41$ & $1.91 \mathrm{E}-41$ \\
\hline mmu-miR-374c-5p & 3.583429 & UP & $1.35 \mathrm{E}-41$ & $2.09 \mathrm{E}-41$ \\
\hline mmu-miR-185-3p & 1.489856 & UP & $1.58 \mathrm{E}-40$ & $2.43 \mathrm{E}-40$ \\
\hline mmu-miR-3058-3p & -1.86908 & DOWN & $2.24 \mathrm{E}-40$ & $3.44 \mathrm{E}-40$ \\
\hline mmu-miR-702-3p & 1.155935 & UP & $1.25 \mathrm{E}-38$ & $1.90 \mathrm{E}-38$ \\
\hline mmu-miR-466e-3p & 2.196258 & UP & $8.59 \mathrm{E}-38$ & $1.30 \mathrm{E}-37$ \\
\hline novel_mir326 & -3.41115 & DOWN & $1.36 \mathrm{E}-37$ & $2.05 \mathrm{E}-37$ \\
\hline mmu-miR-101a-5p & 3.402513 & UP & $2.90 \mathrm{E}-37$ & $4.34 \mathrm{E}-37$ \\
\hline mmu-miR-1a-3p & 1.922464 & UP & $1.04 \mathrm{E}-36$ & $1.55 \mathrm{E}-36$ \\
\hline mmu-miR-466p-3p & 2.029037 & UP & $2.85 \mathrm{E}-36$ & $4.23 \mathrm{E}-36$ \\
\hline mmu-miR-582-3p & 1.315434 & UP & $3.97 \mathrm{E}-36$ & $5.87 \mathrm{E}-36$ \\
\hline mmu-miR-186-3p & 2.51044 & UP & $4.25 \mathrm{E}-35$ & $6.26 \mathrm{E}-35$ \\
\hline novel_mir5 & -1.57375 & DOWN & $8.66 \mathrm{E}-35$ & $1.27 \mathrm{E}-34$ \\
\hline mmu-miR-132-3p & 1.363768 & UP & 4.09E-34 & $5.95 \mathrm{E}-34$ \\
\hline mmu-miR-3968 & 1.134406 & UP & $1.39 \mathrm{E}-33$ & $2.01 \mathrm{E}-33$ \\
\hline novel_mir36 & 1.898365 & UP & $6.36 \mathrm{E}-33$ & $9.14 \mathrm{E}-33$ \\
\hline novel_mir18 & -8.05148 & DOWN & $6.60 \mathrm{E}-33$ & $9.45 \mathrm{E}-33$ \\
\hline mmu-miR-1839-3p & 1.067888 & UP & $3.40 \mathrm{E}-32$ & $4.86 \mathrm{E}-32$ \\
\hline novel_mir86 & -3.17394 & DOWN & $4.13 \mathrm{E}-32$ & $5.86 \mathrm{E}-32$ \\
\hline
\end{tabular}




\begin{tabular}{|c|c|c|c|c|}
\hline mmu-miR-103-1-5p & 2.10117 & UP & $6.16 \mathrm{E}-32$ & 8.73E-32 \\
\hline mmu-miR-7682-3p & -1.38644 & DOWN & $1.84 \mathrm{E}-31$ & $2.60 \mathrm{E}-31$ \\
\hline mmu-miR-155-3p & 3.582296 & UP & 4.55E-31 & $6.39 \mathrm{E}-31$ \\
\hline novel_mir64 & 8.266077 & UP & $5.18 \mathrm{E}-30$ & $7.25 \mathrm{E}-30$ \\
\hline novel_mir188 & -7.86626 & DOWN & $1.24 \mathrm{E}-29$ & $1.73 \mathrm{E}-29$ \\
\hline novel_mir13 & -2.3707 & DOWN & $6.78 \mathrm{E}-29$ & $9.35 \mathrm{E}-29$ \\
\hline mmu-miR-466a-3p & 1.498929 & UP & $6.36 \mathrm{E}-28$ & $8.65 \mathrm{E}-28$ \\
\hline mmu-miR-28c & 3.247326 & UP & $8.66 \mathrm{E}-28$ & $1.17 \mathrm{E}-27$ \\
\hline novel_mir76 & 3.130917 & UP & $9.85 \mathrm{E}-28$ & $1.33 \mathrm{E}-27$ \\
\hline mmu-miR-466f-5p & 2.3157 & UP & $1.08 \mathrm{E}-27$ & $1.45 \mathrm{E}-27$ \\
\hline mmu-miR-6983-3p & -1.37765 & DOWN & $1.48 \mathrm{E}-27$ & $1.98 \mathrm{E}-27$ \\
\hline novel_mir99 & 8.065029 & UP & $7.83 \mathrm{E}-27$ & $1.03 \mathrm{E}-26$ \\
\hline novel_mir178 & -1.99281 & DOWN & $4.81 \mathrm{E}-26$ & $6.32 \mathrm{E}-26$ \\
\hline novel_mir176 & -7.5953 & DOWN & $2.12 \mathrm{E}-25$ & $2.75 \mathrm{E}-25$ \\
\hline novel_mir21 & -7.5468 & DOWN & $1.05 \mathrm{E}-24$ & $1.34 \mathrm{E}-24$ \\
\hline mmu-let-7c-1-3p & -1.06728 & DOWN & $2.13 \mathrm{E}-24$ & $2.72 \mathrm{E}-24$ \\
\hline mmu-miR-6936-5p & -1.5989 & DOWN & $2.63 \mathrm{E}-24$ & 3.34E-24 \\
\hline novel_mir289 & -2.93383 & DOWN & $3.44 \mathrm{E}-24$ & $4.35 \mathrm{E}-24$ \\
\hline mmu-miR-3065-3p & 1.564079 & UP & $3.86 \mathrm{E}-24$ & $4.87 \mathrm{E}-24$ \\
\hline mmu-miR-7091-5p & -1.34692 & DOWN & $4.31 \mathrm{E}-24$ & $5.42 \mathrm{E}-24$ \\
\hline novel_mir161 & -7.47085 & DOWN & $1.17 \mathrm{E}-23$ & $1.47 \mathrm{E}-23$ \\
\hline mmu-miR-5113 & -1.2457 & DOWN & $1.95 \mathrm{E}-23$ & $2.43 \mathrm{E}-23$ \\
\hline novel_mir109 & -2.20537 & DOWN & $2.71 \mathrm{E}-23$ & $3.37 \mathrm{E}-23$ \\
\hline mmu-miR-6988-3p & -1.50746 & DOWN & $3.35 \mathrm{E}-23$ & $4.16 \mathrm{E}-23$ \\
\hline mmu-miR-16-1-3p & 2.57149 & UP & $5.26 \mathrm{E}-23$ & $6.50 \mathrm{E}-23$ \\
\hline mmu-miR-107-5p & -1.1135 & DOWN & $1.05 \mathrm{E}-22$ & $1.30 \mathrm{E}-22$ \\
\hline mmu-miR-3057-3p & -1.78636 & DOWN & $1.06 \mathrm{E}-22$ & $1.30 \mathrm{E}-22$ \\
\hline mmu-miR-3473g & 1.323562 & UP & $2.30 \mathrm{E}-22$ & $2.81 \mathrm{E}-22$ \\
\hline mmu-miR-3109-5p & -1.28262 & DOWN & $3.72 \mathrm{E}-22$ & $4.52 \mathrm{E}-22$ \\
\hline novel_mir191 & -7.32029 & DOWN & $1.06 \mathrm{E}-21$ & $1.28 \mathrm{E}-21$ \\
\hline mmu-miR-184-3p & 1.8 & UP & $2.21 \mathrm{E}-21$ & $2.64 \mathrm{E}-21$ \\
\hline mmu-miR-8120 & -2.25336 & DOWN & $2.44 \mathrm{E}-21$ & 2.91E-21 \\
\hline novel_mir214 & -3.24933 & DOWN & $6.40 \mathrm{E}-21$ & $7.58 \mathrm{E}-21$ \\
\hline novel_mir52 & -7.24629 & DOWN & $8.50 \mathrm{E}-21$ & $1.00 \mathrm{E}-20$ \\
\hline mmu-miR-1b-5p & -1.91251 & DOWN & $1.50 \mathrm{E}-20$ & $1.75 \mathrm{E}-20$ \\
\hline novel_mir145 & -1.19101 & DOWN & $2.69 \mathrm{E}-20$ & $3.14 \mathrm{E}-20$ \\
\hline novel_mir118 & 1.690344 & UP & 4.25E-20 & 4.91E-20 \\
\hline novel_mir10 & 2.952919 & UP & $5.22 \mathrm{E}-20$ & $6.00 \mathrm{E}-20$ \\
\hline
\end{tabular}




\begin{tabular}{|c|c|c|c|c|}
\hline mmu-miR-467d-3p & 1.797493 & UP & $1.35 \mathrm{E}-19$ & $1.54 \mathrm{E}-19$ \\
\hline mmu-miR-30c-2-3p & -1.378 & DOWN & $1.39 \mathrm{E}-19$ & $1.57 \mathrm{E}-19$ \\
\hline novel_mir126 & -7.08583 & DOWN & $5.83 \mathrm{E}-19$ & $6.53 \mathrm{E}-19$ \\
\hline mmu-miR-26a-2-3p & -1.13762 & DOWN & $1.10 \mathrm{E}-18$ & $1.22 \mathrm{E}-18$ \\
\hline novel_mir120 & -7.05148 & DOWN & $1.37 \mathrm{E}-18$ & $1.52 \mathrm{E}-18$ \\
\hline mmu-miR-128-1-5p & -1.01316 & DOWN & $2.19 \mathrm{E}-18$ & $2.41 \mathrm{E}-18$ \\
\hline novel_mir6 & -1.9146 & DOWN & $4.55 \mathrm{E}-18$ & $5.00 \mathrm{E}-18$ \\
\hline novel_mir227 & -6.98022 & DOWN & $7.71 \mathrm{E}-18$ & $8.44 \mathrm{E}-18$ \\
\hline novel_mir290 & 3.113639 & UP & $7.96 \mathrm{E}-18$ & $8.68 \mathrm{E}-18$ \\
\hline mmu-miR-5134-3p & -1.62034 & DOWN & $1.03 \mathrm{E}-17$ & $1.12 \mathrm{E}-17$ \\
\hline mmu-miR-3079-5p & -2.20482 & DOWN & $1.07 \mathrm{E}-17$ & $1.16 \mathrm{E}-17$ \\
\hline novel_mir268 & -1.93459 & DOWN & $1.16 \mathrm{E}-17$ & $1.26 \mathrm{E}-17$ \\
\hline mmu-miR-6418-3p & 1.879378 & UP & $1.17 \mathrm{E}-17$ & $1.26 \mathrm{E}-17$ \\
\hline mmu-miR-3068-3p & 3.665954 & UP & $1.73 \mathrm{E}-17$ & $1.86 \mathrm{E}-17$ \\
\hline novel_mir229 & -6.94322 & DOWN & $1.84 \mathrm{E}-17$ & $1.97 \mathrm{E}-17$ \\
\hline mmu-miR-20b-3p & 4.669337 & UP & $3.07 \mathrm{E}-17$ & $3.27 \mathrm{E}-17$ \\
\hline mmu-miR-34c-5p & 1.397909 & UP & $3.08 \mathrm{E}-17$ & $3.27 \mathrm{E}-17$ \\
\hline mmu-miR-466b-5p & 1.000058 & UP & $5.95 \mathrm{E}-17$ & $6.27 \mathrm{E}-17$ \\
\hline novel_mir187 & 1.576681 & UP & $1.88 \mathrm{E}-16$ & $1.97 \mathrm{E}-16$ \\
\hline mmu-miR-133a-3p & 1.93072 & UP & $1.97 \mathrm{E}-16$ & $2.06 \mathrm{E}-16$ \\
\hline novel_mir143 & 7.181543 & UP & $2.53 \mathrm{E}-16$ & $2.63 \mathrm{E}-16$ \\
\hline mmu-miR-7085-3p & -1.83398 & DOWN & $2.74 \mathrm{E}-16$ & $2.85 \mathrm{E}-16$ \\
\hline mmu-miR-3091-3p & -3.69436 & DOWN & $3.69 \mathrm{E}-16$ & $3.82 \mathrm{E}-16$ \\
\hline novel_mir136 & -6.80572 & DOWN & $3.99 \mathrm{E}-16$ & $4.12 \mathrm{E}-16$ \\
\hline mmu-miR-301a-5p & 1.373193 & UP & $5.57 \mathrm{E}-16$ & $5.74 \mathrm{E}-16$ \\
\hline novel_mir4 & -1.13484 & DOWN & $5.69 \mathrm{E}-16$ & $5.84 \mathrm{E}-16$ \\
\hline novel_mir240 & -6.78496 & DOWN & $6.22 \mathrm{E}-16$ & $6.37 \mathrm{E}-16$ \\
\hline novel_mir59 & -1.75002 & DOWN & $6.39 \mathrm{E}-16$ & $6.53 \mathrm{E}-16$ \\
\hline mmu-let-7b-3p & -1.61385 & DOWN & $6.41 \mathrm{E}-16$ & $6.53 \mathrm{E}-16$ \\
\hline mmu-miR-6537-3p & 2.171559 & UP & $7.93 \mathrm{E}-16$ & $8.04 \mathrm{E}-16$ \\
\hline novel_mir15 & -6.7639 & DOWN & $9.71 \mathrm{E}-16$ & $9.81 \mathrm{E}-16$ \\
\hline mmu-miR-1933-3p & -1.76183 & DOWN & $1.71 \mathrm{E}-15$ & $1.72 \mathrm{E}-15$ \\
\hline mmu-miR-6972-3p & 3.516207 & UP & $1.81 \mathrm{E}-15$ & $1.82 \mathrm{E}-15$ \\
\hline novel_mir204 & 7.07845 & UP & $2.13 \mathrm{E}-15$ & $2.14 \mathrm{E}-15$ \\
\hline mmu-miR-212-5p & -1.1646 & DOWN & $3.34 \mathrm{E}-15$ & $3.33 \mathrm{E}-15$ \\
\hline novel_mir276 & 7.051482 & UP & $3.66 \mathrm{E}-15$ & $3.64 \mathrm{E}-15$ \\
\hline novel_mir168 & -2.51101 & DOWN & $5.53 \mathrm{E}-15$ & $5.48 \mathrm{E}-15$ \\
\hline novel_mir45 & 7.024002 & UP & $6.27 \mathrm{E}-15$ & $6.21 \mathrm{E}-15$ \\
\hline
\end{tabular}




\begin{tabular}{|c|c|c|c|c|}
\hline novel_mir125 & -6.65372 & DOWN & $9.17 \mathrm{E}-15$ & $9.02 \mathrm{E}-15$ \\
\hline novel_mir9 & -1.26692 & DOWN & $1.95 \mathrm{E}-14$ & $1.91 \mathrm{E}-14$ \\
\hline mmu-miR-195a-3p & -2.52729 & DOWN & $2.54 \mathrm{E}-14$ & $2.48 \mathrm{E}-14$ \\
\hline mmu-miR-26a-1-3p & -1.22969 & DOWN & $3.19 \mathrm{E}-14$ & $3.11 \mathrm{E}-14$ \\
\hline novel_mir156 & 6.938272 & UP & $3.21 \mathrm{E}-14$ & $3.12 \mathrm{E}-14$ \\
\hline novel_mir234 & -6.58333 & DOWN & $3.58 \mathrm{E}-14$ & $3.47 \mathrm{E}-14$ \\
\hline mmu-miR-6900-3p & -1.67644 & DOWN & $4.21 \mathrm{E}-14$ & $4.06 \mathrm{E}-14$ \\
\hline novel_mir298 & -6.55908 & DOWN & $5.66 \mathrm{E}-14$ & $5.42 \mathrm{E}-14$ \\
\hline mmu-miR-466m-5p & 1.405504 & UP & $2.59 \mathrm{E}-13$ & $2.46 \mathrm{E}-13$ \\
\hline novel_mir265 & -1.83077 & DOWN & $3.34 \mathrm{E}-13$ & $3.17 \mathrm{E}-13$ \\
\hline mmu-miR-6936-3p & -1.57073 & DOWN & $3.52 \mathrm{E}-13$ & $3.33 \mathrm{E}-13$ \\
\hline novel_mir29 & -6.4578 & DOWN & $3.57 \mathrm{E}-13$ & $3.37 \mathrm{E}-13$ \\
\hline novel_mir220 & -6.43133 & DOWN & $5.68 \mathrm{E}-13$ & $5.33 \mathrm{E}-13$ \\
\hline novel_mir39 & -1.39371 & DOWN & $1.36 \mathrm{E}-12$ & $1.27 \mathrm{E}-12$ \\
\hline mmu-miR-7655-3p & -1.85777 & DOWN & $1.74 \mathrm{E}-12$ & $1.62 \mathrm{E}-12$ \\
\hline mmu-miR-106a-5p & 1.76608 & UP & $3.34 \mathrm{E}-12$ & $3.08 \mathrm{E}-12$ \\
\hline mmu-miR-879-3p & -1.98519 & DOWN & $8.48 \mathrm{E}-12$ & $7.72 \mathrm{E}-12$ \\
\hline novel_mir131 & -6.2614 & DOWN & $9.50 \mathrm{E}-12$ & $8.63 \mathrm{E}-12$ \\
\hline mmu-miR-3076-5p & -2.46651 & DOWN & $9.58 \mathrm{E}-12$ & $8.68 \mathrm{E}-12$ \\
\hline novel_mir287 & -2.27734 & DOWN & $1.00 \mathrm{E}-11$ & $9.08 \mathrm{E}-12$ \\
\hline novel_mir128 & -1.43028 & DOWN & $1.01 \mathrm{E}-11$ & $9.08 \mathrm{E}-12$ \\
\hline mmu-miR-709 & 2.344624 & UP & $1.52 \mathrm{E}-11$ & $1.36 \mathrm{E}-11$ \\
\hline mmu-miR-214-5p & 1.179552 & UP & $1.73 \mathrm{E}-11$ & $1.56 \mathrm{E}-11$ \\
\hline novel_mir288 & -6.2 & DOWN & $2.46 \mathrm{E}-11$ & $2.20 \mathrm{E}-11$ \\
\hline novel_mir37 & -6.2 & DOWN & $2.46 \mathrm{E}-11$ & $2.20 \mathrm{E}-11$ \\
\hline novel_mir201 & 6.533015 & UP & $2.71 \mathrm{E}-11$ & $2.41 \mathrm{E}-11$ \\
\hline novel_mir194 & -2.58333 & DOWN & $2.99 \mathrm{E}-11$ & $2.66 \mathrm{E}-11$ \\
\hline mmu-miR-7652-3p & -2.09148 & DOWN & $3.36 \mathrm{E}-11$ & $2.96 \mathrm{E}-11$ \\
\hline novel_mir292 & -6.13587 & DOWN & $6.42 \mathrm{E}-11$ & $5.66 \mathrm{E}-11$ \\
\hline novel_mir19 & -1.88943 & DOWN & $1.62 \mathrm{E}-10$ & $1.42 \mathrm{E}-10$ \\
\hline novel_mir129 & -2.50426 & DOWN & $2.07 \mathrm{E}-10$ & $1.81 \mathrm{E}-10$ \\
\hline mmu-miR-466m-3p & 2.198031 & UP & $2.23 \mathrm{E}-10$ & $1.94 \mathrm{E}-10$ \\
\hline mmu-miR-129-2-3p & 3.198031 & UP & $2.33 \mathrm{E}-10$ & $2.02 \mathrm{E}-10$ \\
\hline mmu-miR-138-2-3p & -1.18997 & DOWN & $2.66 \mathrm{E}-10$ & $2.30 \mathrm{E}-10$ \\
\hline novel_mir212 & -6.03399 & DOWN & $2.74 \mathrm{E}-10$ & $2.36 \mathrm{E}-10$ \\
\hline novel_mir232 & -1.28649 & DOWN & $2.75 \mathrm{E}-10$ & $2.37 \mathrm{E}-10$ \\
\hline mmu-miR-6954-3p & -1.11606 & DOWN & $3.00 \mathrm{E}-10$ & $2.57 \mathrm{E}-10$ \\
\hline novel_mir171 & -1.17651 & DOWN & $3.47 \mathrm{E}-10$ & $2.95 \mathrm{E}-10$ \\
\hline
\end{tabular}




\begin{tabular}{|c|c|c|c|c|}
\hline novel_mir135 & -5.99837 & DOWN & 4.47E-10 & $3.79 \mathrm{E}-10$ \\
\hline novel_mir34 & -5.99837 & DOWN & $4.47 \mathrm{E}-10$ & $3.79 \mathrm{E}-10$ \\
\hline novel_mir180 & 6.323562 & UP & $5.03 \mathrm{E}-10$ & $4.26 \mathrm{E}-10$ \\
\hline mmu-miR-3061-3p & -1.46303 & DOWN & $5.64 \mathrm{E}-10$ & $4.76 \mathrm{E}-10$ \\
\hline novel_mir67 & -5.96184 & DOWN & 7.29E-10 & $6.11 \mathrm{E}-10$ \\
\hline mmu-miR-1955-3p & 2.349097 & UP & 8.32E-10 & $6.97 \mathrm{E}-10$ \\
\hline mmu-miR-6915-5p & -2.44197 & DOWN & $8.73 \mathrm{E}-10$ & $7.29 \mathrm{E}-10$ \\
\hline novel_mir53 & 6.277758 & UP & $9.11 \mathrm{E}-10$ & $7.59 \mathrm{E}-10$ \\
\hline mmu-miR-6960-5p & -1.28888 & DOWN & $1.51 \mathrm{E}-09$ & $1.25 \mathrm{E}-09$ \\
\hline novel_mir242 & -5.88589 & DOWN & $1.95 \mathrm{E}-09$ & $1.61 \mathrm{E}-09$ \\
\hline novel_mir246 & -5.88589 & DOWN & $1.95 \mathrm{E}-09$ & $1.61 \mathrm{E}-09$ \\
\hline novel_mir269 & -5.84636 & DOWN & $3.20 \mathrm{E}-09$ & $2.62 \mathrm{E}-09$ \\
\hline novel_mir196 & -5.84636 & DOWN & $3.20 \mathrm{E}-09$ & $2.62 \mathrm{E}-09$ \\
\hline mmu-miR-7053-3p & -2.16582 & DOWN & $3.41 \mathrm{E}-09$ & $2.78 \mathrm{E}-09$ \\
\hline mmu-miR-1b-3p & 1.145919 & UP & 4.02E-09 & $3.27 \mathrm{E}-09$ \\
\hline mmu-miR-3079-3p & -1.3587 & DOWN & 4.54E-09 & $3.68 \mathrm{E}-09$ \\
\hline novel_mir235 & -1.73784 & DOWN & $4.71 \mathrm{E}-09$ & $3.81 \mathrm{E}-09$ \\
\hline mmu-miR-19a-5p & 4.230453 & UP & 7.08E-09 & $5.69 \mathrm{E}-09$ \\
\hline mmu-miR-8090 & 1.350529 & UP & 7.66E-09 & $6.14 \mathrm{E}-09$ \\
\hline novel_mir170 & -5.7639 & DOWN & 8.69E-09 & $6.89 \mathrm{E}-09$ \\
\hline novel_mir203 & -5.7639 & DOWN & 8.69E-09 & $6.89 \mathrm{E}-09$ \\
\hline novel_mir40 & -5.7639 & DOWN & 8.69E-09 & 6.89E-09 \\
\hline novel_mir258 & -5.7639 & DOWN & 8.69E-09 & $6.89 \mathrm{E}-09$ \\
\hline novel_mir306 & -5.7639 & DOWN & 8.69E-09 & $6.89 \mathrm{E}-09$ \\
\hline mmu-miR-547-3p & 2.295548 & UP & $1.02 \mathrm{E}-08$ & 8.08E-09 \\
\hline mmu-miR-201-5p & 1.123263 & UP & $1.13 \mathrm{E}-08$ & $8.95 \mathrm{E}-09$ \\
\hline mmu-miR-7657-3p & 4.181543 & UP & $1.40 \mathrm{E}-08$ & $1.10 \mathrm{E}-08$ \\
\hline novel_mir127 & -5.72083 & DOWN & $1.44 \mathrm{E}-08$ & $1.13 \mathrm{E}-08$ \\
\hline novel_mir224 & -5.72083 & DOWN & $1.44 \mathrm{E}-08$ & $1.13 \mathrm{E}-08$ \\
\hline mmu-miR-3964 & 2.579902 & UP & $1.84 \mathrm{E}-08$ & $1.44 \mathrm{E}-08$ \\
\hline novel_mir20 & 6.024002 & UP & $1.87 \mathrm{E}-08$ & $1.45 \mathrm{E}-08$ \\
\hline mmu-miR-375-3p & -1.16548 & DOWN & $2.15 \mathrm{E}-08$ & $1.66 \mathrm{E}-08$ \\
\hline novel_mir12 & -1.07571 & DOWN & $2.16 \mathrm{E}-08$ & $1.67 \mathrm{E}-08$ \\
\hline novel_mir302 & -5.67644 & DOWN & $2.38 \mathrm{E}-08$ & $1.83 \mathrm{E}-08$ \\
\hline mmu-miR-8103 & -1.42187 & DOWN & $3.47 \mathrm{E}-08$ & $2.67 \mathrm{E}-08$ \\
\hline novel_mir24 & -2.23715 & DOWN & $5.93 \mathrm{E}-08$ & 4.52E-08 \\
\hline novel_mir317 & 5.908525 & UP & $6.41 \mathrm{E}-08$ & 4.88E-08 \\
\hline novel_mir219 & -5.58333 & DOWN & $6.57 \mathrm{E}-08$ & $5.00 \mathrm{E}-08$ \\
\hline
\end{tabular}




\begin{tabular}{|c|c|c|c|c|}
\hline mmu-miR-7042-5p & -1.65995 & DOWN & $6.73 \mathrm{E}-08$ & $5.10 \mathrm{E}-08$ \\
\hline mmu-let-7g-3p & 1.222279 & UP & $6.89 \mathrm{E}-08$ & $5.21 \mathrm{E}-08$ \\
\hline novel_mir304 & 2.393951 & UP & 9.91E-08 & $7.46 \mathrm{E}-08$ \\
\hline mmu-miR-6908-5p & -2.81394 & DOWN & 9.91E-08 & $7.46 \mathrm{E}-08$ \\
\hline novel_mir139 & -5.53442 & DOWN & $1.10 \mathrm{E}-07$ & $8.23 \mathrm{E}-08$ \\
\hline novel_mir309 & 5.847124 & UP & $1.20 \mathrm{E}-07$ & $8.96 \mathrm{E}-08$ \\
\hline mmu-miR-6900-5p & -1.84636 & DOWN & $1.23 \mathrm{E}-07$ & $9.21 \mathrm{E}-08$ \\
\hline novel_mir14 & 1.834524 & UP & $1.60 \mathrm{E}-07$ & $1.19 \mathrm{E}-07$ \\
\hline mmu-miR-6979-3p & -1.7788 & DOWN & 2.09E-07 & $1.55 \mathrm{E}-07$ \\
\hline mmu-miR-7059-5p & -1.91348 & DOWN & $2.14 \mathrm{E}-07$ & $1.58 \mathrm{E}-07$ \\
\hline novel_mir286 & 5.782994 & UP & $2.24 \mathrm{E}-07$ & $1.65 \mathrm{E}-07$ \\
\hline mmu-miR-7677-3p & -2.58333 & DOWN & 2.62E-07 & $1.93 \mathrm{E}-07$ \\
\hline mmu-miR-3100-5p & -1.35996 & DOWN & $2.71 \mathrm{E}-07$ & $1.99 \mathrm{E}-07$ \\
\hline novel_mir81 & -5.43133 & DOWN & $3.08 \mathrm{E}-07$ & $2.25 \mathrm{E}-07$ \\
\hline novel_mir25 & 2.323562 & UP & $3.24 \mathrm{E}-07$ & $2.36 \mathrm{E}-07$ \\
\hline mmu-miR-3102-5p.2-5p & -1.50832 & DOWN & $3.52 \mathrm{E}-07$ & $2.55 \mathrm{E}-07$ \\
\hline novel_mir185 & -1.93019 & DOWN & $4.27 \mathrm{E}-07$ & $3.09 \mathrm{E}-07$ \\
\hline mmu-miR-7655-5p & -2.92437 & DOWN & $4.45 \mathrm{E}-07$ & $3.21 \mathrm{E}-07$ \\
\hline novel_mir181 & -1.28787 & DOWN & $5.09 \mathrm{E}-07$ & $3.66 \mathrm{E}-07$ \\
\hline novel_mir213 & -5.37688 & DOWN & 5.17E-07 & $3.70 \mathrm{E}-07$ \\
\hline novel_mir271 & -5.37688 & DOWN & $5.17 \mathrm{E}-07$ & $3.70 \mathrm{E}-07$ \\
\hline novel_mir329 & -5.37688 & DOWN & $5.17 \mathrm{E}-07$ & $3.70 \mathrm{E}-07$ \\
\hline mmu-miR-7043-5p & 1.600402 & UP & $5.56 \mathrm{E}-07$ & 3.97E-07 \\
\hline mmu-miR-34b-5p & 1.360088 & UP & $5.80 \mathrm{E}-07$ & 4.13E-07 \\
\hline mmu-miR-181b-1-3p & 1.803555 & UP & $5.88 \mathrm{E}-07$ & $4.17 \mathrm{E}-07$ \\
\hline novel_mir58 & -1.50087 & DOWN & 7.07E-07 & $5.01 \mathrm{E}-07$ \\
\hline novel_mir313 & 5.64549 & UP & 7.97E-07 & $5.63 \mathrm{E}-07$ \\
\hline novel_mir252 & -5.32029 & DOWN & $8.72 \mathrm{E}-07$ & $6.11 \mathrm{E}-07$ \\
\hline novel_mir300 & -5.32029 & DOWN & $8.72 \mathrm{E}-07$ & $6.11 \mathrm{E}-07$ \\
\hline novel_mir179 & -5.32029 & DOWN & $8.72 \mathrm{E}-07$ & $6.11 \mathrm{E}-07$ \\
\hline mmu-miR-6994-3p & -1.61834 & DOWN & $1.20 \mathrm{E}-06$ & $8.36 \mathrm{E}-07$ \\
\hline novel_mir151 & -5.2614 & DOWN & $1.47 \mathrm{E}-06$ & $1.02 \mathrm{E}-06$ \\
\hline mmu-miR-6985-3p & -1.32215 & DOWN & $1.48 \mathrm{E}-06$ & $1.03 \mathrm{E}-06$ \\
\hline mmu-miR-497b & -1.44197 & DOWN & $1.48 \mathrm{E}-06$ & $1.03 \mathrm{E}-06$ \\
\hline mmu-miR-1231-3p & -1.85864 & DOWN & $1.52 \mathrm{E}-06$ & $1.05 \mathrm{E}-06$ \\
\hline novel_mir198 & -1.56396 & DOWN & $1.85 \mathrm{E}-06$ & $1.27 \mathrm{E}-06$ \\
\hline mmu-miR-6395 & 1.942472 & UP & $2.24 \mathrm{E}-06$ & $1.54 \mathrm{E}-06$ \\
\hline novel_mir35 & -5.2 & DOWN & $2.50 \mathrm{E}-06$ & $1.70 \mathrm{E}-06$ \\
\hline
\end{tabular}




\begin{tabular}{|c|c|c|c|c|}
\hline novel_mir123 & -5.2 & DOWN & $2.50 \mathrm{E}-06$ & $1.70 \mathrm{E}-06$ \\
\hline novel_mir241 & -5.2 & DOWN & $2.50 \mathrm{E}-06$ & $1.70 \mathrm{E}-06$ \\
\hline novel_mir69 & -5.2 & DOWN & $2.50 \mathrm{E}-06$ & $1.70 \mathrm{E}-06$ \\
\hline novel_mir138 & -5.2 & DOWN & $2.50 \mathrm{E}-06$ & $1.70 \mathrm{E}-06$ \\
\hline novel_mir275 & 5.493487 & UP & $2.89 \mathrm{E}-06$ & $1.96 \mathrm{E}-06$ \\
\hline novel_mir209 & 5.493487 & UP & 2.89E-06 & $1.96 \mathrm{E}-06$ \\
\hline mmu-miR-7669-5p & 1.034055 & UP & 2.99E-06 & $2.02 \mathrm{E}-06$ \\
\hline mmu-miR-6992-5p & -3.04567 & DOWN & 3.32E-06 & $2.23 \mathrm{E}-06$ \\
\hline mmu-miR-7037-5p & -1.19491 & DOWN & $3.33 \mathrm{E}-06$ & $2.24 \mathrm{E}-06$ \\
\hline mmu-miR-3085-3p & 2.596581 & UP & $3.54 \mathrm{E}-06$ & 2.37E-06 \\
\hline mmu-miR-1191b-3p & -2.2614 & DOWN & 4.14E-06 & $2.76 \mathrm{E}-06$ \\
\hline novel_mir100 & -5.13587 & DOWN & 4.25E-06 & $2.83 \mathrm{E}-06$ \\
\hline novel_mir41 & -1.70028 & DOWN & 4.27E-06 & $2.84 \mathrm{E}-06$ \\
\hline mmu-miR-489-3p & 5.411025 & UP & $5.56 \mathrm{E}-06$ & $3.68 \mathrm{E}-06$ \\
\hline novel_mir190 & -5.06876 & DOWN & $7.25 \mathrm{E}-06$ & 4.78E-06 \\
\hline novel_mir280 & -5.06876 & DOWN & $7.25 \mathrm{E}-06$ & $4.78 \mathrm{E}-06$ \\
\hline mmu-miR-5622-3p & -1.48379 & DOWN & $8.10 \mathrm{E}-06$ & $5.32 \mathrm{E}-06$ \\
\hline mmu-miR-450a-1-3p & -1.15349 & DOWN & $8.35 \mathrm{E}-06$ & $5.47 \mathrm{E}-06$ \\
\hline mmu-miR-130a-5p & 4.411025 & UP & $1.06 \mathrm{E}-05$ & $6.93 \mathrm{E}-06$ \\
\hline mmu-miR-5134-5p & -1.29659 & DOWN & $1.15 \mathrm{E}-05$ & $7.48 \mathrm{E}-06$ \\
\hline novel_mir144 & -4.99837 & DOWN & $1.24 \mathrm{E}-05$ & $8.01 \mathrm{E}-06$ \\
\hline novel_mir231 & -4.99837 & DOWN & $1.24 \mathrm{E}-05$ & 8.01E-06 \\
\hline novel_mir205 & -4.99837 & DOWN & $1.24 \mathrm{E}-05$ & 8.01E-06 \\
\hline mmu-miR-1930-3p & -1.10094 & DOWN & $1.95 \mathrm{E}-05$ & $1.24 \mathrm{E}-05$ \\
\hline mmu-miR-144-5p & 5.230453 & UP & $2.08 \mathrm{E}-05$ & $1.31 \mathrm{E}-05$ \\
\hline novel_mir173 & 5.230453 & UP & $2.08 \mathrm{E}-05$ & $1.31 \mathrm{E}-05$ \\
\hline novel_mir55 & 5.230453 & UP & $2.08 \mathrm{E}-05$ & $1.31 \mathrm{E}-05$ \\
\hline novel_mir172 & 5.230453 & UP & $2.08 \mathrm{E}-05$ & $1.31 \mathrm{E}-05$ \\
\hline mmu-miR-467e-3p & 3.493487 & UP & $2.58 \mathrm{E}-05$ & $1.63 \mathrm{E}-05$ \\
\hline novel_mir158 & -1.67644 & DOWN & $2.64 \mathrm{E}-05$ & $1.66 \mathrm{E}-05$ \\
\hline novel_mir141 & -1.62397 & DOWN & $2.83 \mathrm{E}-05$ & $1.78 \mathrm{E}-05$ \\
\hline mmu-miR-6970-3p & 1.764135 & UP & $3.10 \mathrm{E}-05$ & $1.95 \mathrm{E}-05$ \\
\hline mmu-miR-7654-3p & -1.2614 & DOWN & $3.16 \mathrm{E}-05$ & $1.97 \mathrm{E}-05$ \\
\hline mmu-miR-194-2-3p & -1.70619 & DOWN & $3.61 \mathrm{E}-05$ & $2.26 \mathrm{E}-05$ \\
\hline novel_mir316 & -4.84636 & DOWN & $3.67 \mathrm{E}-05$ & $2.29 \mathrm{E}-05$ \\
\hline mmu-miR-7012-5p & -1.89883 & DOWN & $3.85 \mathrm{E}-05$ & $2.39 \mathrm{E}-05$ \\
\hline mmu-miR-8106 & -1.10174 & DOWN & 4.09E-05 & $2.53 \mathrm{E}-05$ \\
\hline mmu-miR-7075-3p & -1.04101 & DOWN & $4.12 \mathrm{E}-05$ & $2.55 \mathrm{E}-05$ \\
\hline
\end{tabular}




\begin{tabular}{|c|c|c|c|c|}
\hline mmu-miR-135b-5p & 1.908525 & UP & $4.21 \mathrm{E}-05$ & $2.60 \mathrm{E}-05$ \\
\hline mmu-miR-504-5p & -2.53442 & DOWN & 4.32E-05 & $2.66 \mathrm{E}-05$ \\
\hline mmu-miR-7036b-3p & -1.81394 & DOWN & $4.41 \mathrm{E}-05$ & $2.71 \mathrm{E}-05$ \\
\hline mmu-miR-7656-3p & -2.79192 & DOWN & $4.51 \mathrm{E}-05$ & $2.77 \mathrm{E}-05$ \\
\hline mmu-miR-6978-3p & 2.986527 & UP & $5.47 \mathrm{E}-05$ & $3.35 \mathrm{E}-05$ \\
\hline mmu-miR-1190 & 2.216647 & UP & $6.25 \mathrm{E}-05$ & $3.82 \mathrm{E}-05$ \\
\hline novel_mir301 & -4.7639 & DOWN & $6.35 \mathrm{E}-05$ & $3.85 \mathrm{E}-05$ \\
\hline novel_mir328 & -4.7639 & DOWN & $6.35 \mathrm{E}-05$ & $3.85 \mathrm{E}-05$ \\
\hline novel_mir226 & -4.7639 & DOWN & $6.35 \mathrm{E}-05$ & $3.85 \mathrm{E}-05$ \\
\hline mmu-miR-6986-5p & -1.2614 & DOWN & $6.37 \mathrm{E}-05$ & $3.85 \mathrm{E}-05$ \\
\hline mmu-miR-5128 & -1.14459 & DOWN & $7.03 \mathrm{E}-05$ & $4.25 \mathrm{E}-05$ \\
\hline mmu-miR-7676-3p & 1.125623 & UP & 7.14E-05 & $4.31 \mathrm{E}-05$ \\
\hline mmu-miR-149-3p & 1.86413 & UP & 7.19E-05 & $4.33 \mathrm{E}-05$ \\
\hline novel_mir183 & -1.54151 & DOWN & 8.94E-05 & 5.37E-05 \\
\hline novel_mir166 & -4.67644 & DOWN & 0.000110237 & $6.58 \mathrm{E}-05$ \\
\hline novel_mir266 & -4.67644 & DOWN & 0.000110237 & $6.58 \mathrm{E}-05$ \\
\hline novel_mir77 & -4.67644 & DOWN & 0.000110237 & $6.58 \mathrm{E}-05$ \\
\hline novel_mir314 & -4.67644 & DOWN & 0.000110237 & $6.58 \mathrm{E}-05$ \\
\hline mmu-miR-3098-5p & 2.160063 & UP & 0.000112108 & $6.68 \mathrm{E}-05$ \\
\hline novel_mir303 & -1.61504 & DOWN & 0.000118633 & $7.05 \mathrm{E}-05$ \\
\hline mmu-miR-7002-5p & -3.06876 & DOWN & 0.00012252 & 7.27E-05 \\
\hline mmu-miR-7008-5p & -2.67644 & DOWN & 0.000126479 & 7.49E-05 \\
\hline novel_mir320 & -1.43133 & DOWN & 0.000135964 & 8.03E-05 \\
\hline mmu-miR-9769-3p & -1.71206 & DOWN & 0.00015198 & 8.92E-05 \\
\hline novel_mir163 & 4.908525 & UP & 0.000158647 & $9.27 \mathrm{E}-05$ \\
\hline novel_mir200 & 4.908525 & UP & 0.000158647 & $9.27 \mathrm{E}-05$ \\
\hline novel_mir184 & 4.908525 & UP & 0.000158647 & $9.27 \mathrm{E}-05$ \\
\hline mmu-miR-128-2-5p & 1.859615 & UP & 0.000161247 & $9.40 \mathrm{E}-05$ \\
\hline novel_mir82 & -1.64327 & DOWN & 0.000164637 & $9.59 \mathrm{E}-05$ \\
\hline novel_mir174 & -4.58333 & DOWN & 0.000192065 & 0.000111304 \\
\hline mmu-miR-3082-5p & -1.2614 & DOWN & 0.000261996 & 0.000151122 \\
\hline mmu-miR-6904-5p & -1.28649 & DOWN & 0.000268023 & 0.000154358 \\
\hline novel_mir278 & 4.782994 & UP & 0.000316589 & 0.000180367 \\
\hline novel_mir91 & 4.782994 & UP & 0.000316589 & 0.000180367 \\
\hline novel_mir291 & -4.48379 & DOWN & 0.000335973 & 0.000189375 \\
\hline novel_mir293 & -4.48379 & DOWN & 0.000335973 & 0.000189375 \\
\hline novel_mir66 & -4.48379 & DOWN & 0.000335973 & 0.000189375 \\
\hline novel_mir48 & -4.48379 & DOWN & 0.000335973 & 0.000189375 \\
\hline
\end{tabular}




\begin{tabular}{|c|c|c|c|c|}
\hline novel_mir272 & -4.48379 & DOWN & 0.000335973 & 0.000189375 \\
\hline novel_mir239 & -4.48379 & DOWN & 0.000335973 & 0.000189375 \\
\hline mmu-miR-7072-5p & 3.908525 & UP & 0.000347975 & 0.000195842 \\
\hline mmu-miR-5622-5p & 1.323562 & UP & 0.000390572 & 0.000219483 \\
\hline mmu-miR-6993-5p & -2.1094 & DOWN & 0.000407456 & 0.000228625 \\
\hline mmu-miR-7035-5p & 1.504134 & UP & 0.000440736 & 0.000246924 \\
\hline mmu-miR-6964-3p & -2.2614 & DOWN & 0.000500829 & 0.000280169 \\
\hline mmu-miR-3077-3p & 1.05226 & UP & 0.000515207 & 0.000287777 \\
\hline mmu-miR-7046-5p & -1.53893 & DOWN & 0.000529398 & 0.000295259 \\
\hline novel_mir230 & -1.48379 & DOWN & 0.000546959 & 0.000304596 \\
\hline mmu-miR-6953-3p & -1.11384 & DOWN & 0.000577982 & 0.000321389 \\
\hline novel_mir257 & -4.37688 & DOWN & 0.000590155 & 0.00032475 \\
\hline novel_mir149 & -4.37688 & DOWN & 0.000590155 & 0.00032475 \\
\hline novel_mir331 & -4.37688 & DOWN & 0.000590155 & 0.00032475 \\
\hline novel_mir134 & -4.37688 & DOWN & 0.000590155 & 0.00032475 \\
\hline novel_mir299 & -4.37688 & DOWN & 0.000590155 & 0.00032475 \\
\hline novel_mir162 & -4.37688 & DOWN & 0.000590155 & 0.00032475 \\
\hline novel_mir210 & -4.37688 & DOWN & 0.000590155 & 0.00032475 \\
\hline mmu-miR-669m-3p & 1.975639 & UP & 0.000621971 & 0.000340741 \\
\hline novel_mir211 & 4.64549 & UP & 0.000636785 & 0.000347318 \\
\hline novel_mir84 & 4.64549 & UP & 0.000636785 & 0.000347318 \\
\hline novel_mir114 & 4.64549 & UP & 0.000636785 & 0.000347318 \\
\hline novel_mir195 & -1.1874 & DOWN & 0.000693046 & 0.000376895 \\
\hline mmu-miR-6915-3p & -1.23443 & DOWN & 0.0007433 & 0.000403043 \\
\hline novel_mir148 & -1.56396 & DOWN & 0.000744933 & 0.000403339 \\
\hline mmu-miR-6948-3p & -1.2614 & DOWN & 0.000764455 & 0.000412959 \\
\hline novel_mir60 & -1.50241 & DOWN & 0.000774286 & 0.000417232 \\
\hline mmu-miR-6399 & -1.89883 & DOWN & 0.000776218 & 0.000417232 \\
\hline mmu-miR-7671-3p & -1.89883 & DOWN & 0.000776218 & 0.000417232 \\
\hline mmu-miR-7119-5p & -3.48379 & DOWN & 0.000922108 & 0.000494218 \\
\hline mmu-miR-7003-5p & -2.4134 & DOWN & 0.000958326 & 0.000512889 \\
\hline mmu-miR-6919-3p & -2.7639 & DOWN & 0.001032767 & 0.00054851 \\
\hline novel_mir133 & -4.2614 & DOWN & 0.001041152 & 0.00054851 \\
\hline novel_mir259 & -4.2614 & DOWN & 0.001041152 & 0.00054851 \\
\hline novel_mir7 & -4.2614 & DOWN & 0.001041152 & 0.00054851 \\
\hline novel_mir43 & -4.2614 & DOWN & 0.001041152 & 0.00054851 \\
\hline novel_mir83 & -4.2614 & DOWN & 0.001041152 & 0.00054851 \\
\hline novel_mir274 & -4.2614 & DOWN & 0.001041152 & 0.00054851 \\
\hline
\end{tabular}




\begin{tabular}{|c|c|c|c|c|}
\hline novel_mir261 & -4.2614 & DOWN & 0.001041152 & 0.00054851 \\
\hline mmu-miR-465c-3p & -4.2614 & DOWN & 0.001041152 & 0.00054851 \\
\hline novel_mir282 & -4.2614 & DOWN & 0.001041152 & 0.00054851 \\
\hline mmu-miR-7029-3p & -1.59398 & DOWN & 0.001046122 & 0.000550347 \\
\hline mmu-miR-7036a-3p & -1.23283 & DOWN & 0.001064539 & 0.000559242 \\
\hline mmu-miR-6987-5p & -1.84636 & DOWN & 0.001200819 & 0.000629943 \\
\hline mmu-miR-297c-3p & 2.545954 & UP & 0.001439253 & 0.000752894 \\
\hline mmu-miR-879-5p & 2.908525 & UP & 0.001549868 & 0.000809617 \\
\hline mmu-miR-126b-5p & 1.157552 & UP & 0.001611301 & 0.000838166 \\
\hline mmu-miR-7057-3p & -2.67644 & DOWN & 0.001749209 & 0.000907359 \\
\hline novel_mir49 & -4.13587 & DOWN & 0.001845165 & 0.000941334 \\
\hline novel_mir38 & -4.13587 & DOWN & 0.001845165 & 0.000941334 \\
\hline novel_mir312 & -4.13587 & DOWN & 0.001845165 & 0.000941334 \\
\hline novel_mir78 & -4.13587 & DOWN & 0.001845165 & 0.000941334 \\
\hline novel_mir279 & -4.13587 & DOWN & 0.001845165 & 0.000941334 \\
\hline novel_mir285 & -4.13587 & DOWN & 0.001845165 & 0.000941334 \\
\hline novel_mir79 & -4.13587 & DOWN & 0.001845165 & 0.000941334 \\
\hline novel_mir97 & -4.13587 & DOWN & 0.001845165 & 0.000941334 \\
\hline novel_mir80 & -4.13587 & DOWN & 0.001845165 & 0.000941334 \\
\hline novel_mir169 & -4.13587 & DOWN & 0.001845165 & 0.000941334 \\
\hline novel_mir104 & -4.13587 & DOWN & 0.001845165 & 0.000941334 \\
\hline
\end{tabular}

Table S4. Primers used for real time PCR

\begin{tabular}{|ll|}
\hline Primers & Sequences (' $\mathbf{5}^{\prime} \mathbf{t o} \mathbf{}^{\prime}$ ) \\
\hline Mitf-F & AACCGACAGAAGAAGCTGGA \\
\hline Mitf-R & TGATGATCCGTTCACCAGA \\
\hline Spi1-F & GAGAAGCTGATGGCTTGGAG \\
\hline Spi1-R & GCTTGGACGAGAACTGGAAG \\
\hline Socs1-F & CTGCGGCTTCTATTGGGGAC \\
\hline Socs1-R & AAAAGGCAGTCGAAGGTCTCG \\
\hline GAPDH-F & CAATGAATAGGGCTACAGCA \\
\hline GAPDH-R & AGGGAGATGCTCAGTGTTGG \\
\hline miR-155 & TTAATGCTAATTGTGATAGGGGT \\
\hline
\end{tabular}

